\title{
Effect of dietary interventions in mild cognitive impairment: a systematic review
}

\author{
Andrea M. McGrattan ${ }^{1 *}$, Claire T. McEvoy ${ }^{1,2}$, Bernadette McGuinness ${ }^{1}$, Michelle C. McKinley ${ }^{1}$ and \\ Jayne V. Woodside ${ }^{1}$ \\ ${ }^{1}$ Centre for Public Health, Queen's University Belfast, Belfast BT12 6BJ, UK \\ ${ }^{2}$ Global Brain Health Institute, University of California San Francisco, 4150 Clement Street, Veterans Affairs Medical Center \\ (116-H), San Francisco, CA 94121, USA
}

(Submitted 19 December 2017 - Final revision received 17 August 2018 - Accepted 8 September 2018)

\section{Abstract}

Diet has been investigated in relation to its ability to promote cognitive function. However, evidence is currently limited and has rarely been systematically reviewed, particularly in a mild cognitive impairment (MCI) population. This review examined the effect of diet on cognitive outcomes in MCI patients. A total of five databases were searched to find randomised controlled trial (RCT) studies, with diet as the main focus, in MCI participants. The primary outcome was incident dementia and/or Alzheimer's disease (AD) and secondary outcomes included cognitive function across different domains using validated neuropsychological tests. Sixteen studies met the inclusion criteria. There was a high degree of heterogeneity relating to the nature of the dietary intervention and cognitive outcomes measured, thus making study comparisons difficult. Supplementation with vitamin E (one study, $n$ 516), ginkgo biloba (one study, $n$ 482) or Fortasyn Connect (one study, $n$ 311) had no significant effect on progression from MCI to dementia and/or AD. For cognitive function, the findings showed some improvements in performance, particularly in memory, with the most consistent results shown by B vitamins, including folic acid (one study, $n$ 266), folic acid alone (one study, $n$ 180), DHA and EPA (two studies, $n 36$ and $n$ 86), DHA (one study, $n$ 240) and flavonol supplementation (one study, $n$ 90). The findings indicate that dietary factors may have a potential benefit for cognitive function in MCI patients. Further welldesigned trials are needed, with standardised and robust measures of cognition to investigate the influence of diet on cognitive status.

\section{Key words: Diet: Lifestyle: Interventions: Mild cognitive impairment: Systematic reviews}

Cognitive impairment poses a major global public health challenge due to increasing prevalence in line with population ageing $^{(1)}$. The transition from mild cognitive impairment (MCI) through to the various forms of dementia, such as Alzheimer's disease $(\mathrm{AD})$, is one of the costliest burdens on health service delivery $^{(2)}$. The National Institute for Aging-Alzheimer's Association (NIA-AA) developed core clinical criteria to inform the diagnosis of $\mathrm{MCI}^{(3)}$. This identifies that a person with MCI should display a change in cognition, expressed through personal concern or identification from a physician. In addition, individuals should display a lower performance in at least one cognitive domain than that expected for their age and education, over a period of time. Such domains are memory, executive function, attention, visuospatial skills and language. Finally, individuals with MCI may have slight problems with complex daily tasks, however, generally live an independent lifestyle with minimal assistance ${ }^{(3)}$. MCI is described as a transitional stage between the expected cognitive decline of normal ageing and that of dementia ${ }^{(4)}$. Furthermore, it has been estimated that $46 \%$ of MCI patients develop dementia within 3 years from diagnosis ${ }^{(5)}$. Therefore, it is critical to identify effective interventions that can protect against cognitive decline in this vulnerable high risk group ${ }^{(6)}$.

Despite pharmacological advances, there are no effective treatments to delay or reverse cognitive impairment. The inflammatory mechanisms and oxidative stress involved in the aetiology of cognitive decline and $\operatorname{dementia}^{(7)}$, indicates a potential role for nutrition in its prevention ${ }^{(8)}$. Furthermore, processes such as neurogenesis and neuronal connectivity involved in the function of the brain are influenced by dietary components $^{(9,10)}$. The role of nutrition in cognitive health outcomes has been examined in terms of a range of nutrients/ dietary patterns, investigating the role that single nutrients, such as $n$-3 PUFA $^{(7)}$, as well as whole foods/diet interventions, such as the Dietary Approaches to Stop Hypertension (DASH) $\operatorname{diet}^{(11)}$, a ketogenic diet ${ }^{(12)}$ or the Mediterranean $\operatorname{diet}^{(13)}$ may

Abbreviations: AD, Alzheimer's disease; GI, glycaemic index; HF, high flavonol; HR, hazard ratio; LF, low flavonol; MCI, mild cognitive impairment; MMSE, Mini Mental State Examination; RCT, randomised controlled trial.

* Corresponding author: A. M. McGrattan, email amcgrattan02@qub.ac.uk 
have, particularly in relation to their effect on reducing inflammation and oxidative stress ${ }^{(14-16)}$. It has been suggested that, although investigations into single nutrients have importance from a mechanistic point of view, studies which provide whole-diet analysis acknowledge that, in everyday situations, foods are consumed in complex combinations and may be a more representative approach to measure the effect of diet on cognition ${ }^{(17)}$. Furthermore, ensuring older adults with MCI stay physically active could have beneficial effects on cognition $^{(18,19)}$, alongside engaging in cognitive training strategies to boost cognitive function. This involves a variety of either computerised or hand-written techniques to enhance memory, language and attention ${ }^{(20)}$. However, the available research in this area is variable, with a lack of specific studies in $\mathrm{MCI}^{(6)}$.

Ultimately, there is a need for this systematic review to examine what is known to date about the role of diet on cognitive health, either independently or in conjunction with other lifestyle modifications, specifically in a MCI population. To our knowledge, the effect of dietary interventions on cognitive health outcomes, particularly in high risk populations, like MCI has not been previously systematically reviewed and therefore this has the potential to establish the evidence base for possible management strategies and also define the scope for future research, if required. Thus, the aim of this systematic review was to examine the effect of diet, either alone or in combination with lifestyle and/or cognitive strategies, on cognitive health outcomes in patients with MCI.

\section{Methods}

The methods for this systematic review were based on the Centre for Reviews and Dissemination guidance for undertaking systematic reviews in health care ${ }^{(21)}$ and the review protocol was registered with PROSPERO (PROSPERO 2017: CRD 42017067267). To be included in this review, the article had to be a randomised controlled trial (RCT) design, conducted in patients with MCI and with diet as the main focus of the intervention. Pilot studies were excluded when a paper clearly stated that the research was a 'pilot study'. Interventions could focus on diet alone (a dietary pattern or dietary supplements) or in combination with lifestyle and/or cognitive strategies. An overview of the inclusion and exclusion criteria is provided in Table 1 . Incident dementia or AD was the primary outcome measure. Secondary outcomes included overall cognitive function or specific cognitive domains such as memory, executive function, language, attention or visuospatial skills measured using validated neuropsychological tests, for example, Mini Mental State Examination (MMSE), Cambridge Cognition Examination or Repeatable Battery for the Assessment of Neuropsychological Status.

\section{Study identification}

A comprehensive literature search was undertaken in June 2016 using Ovid MEDLINE, EMBASE, PsycINFO, Web of Science and Scopus. A suitable search strategy was devised considering key terms used in associated reviews relating to 'diet', 'lifestyle', 'cognitive strategies', 'cognition' and 'behaviour change'. Studies were restricted to English Language and similar search terms were used in each database. This detailed search strategy was developed in Ovid MEDLINE (online Supplementary Table S1) and this strategy was tailored for the other databases. The literature search was repeated in November 2016 and March 2018 to identify new publications. The reference lists of articles and other relevant systematic reviews were screened for potential trials not identified by the electronic search.

\section{Data extraction}

Titles and abstracts of potentially eligible studies were screened by the first author (A. M. M.). Any articles not meeting the inclusion criteria were excluded at this stage. Full text articles were obtained for the remaining studies and the study methodology was further assessed for eligibility (A. M. M.). Any queries with regards to inclusion of articles were

Table 1. An overview of the inclusion and exclusion criteria for this systematic review

\begin{tabular}{|c|c|c|}
\hline & Inclusion criteria & Exclusion criteria \\
\hline Study design & Randomised controlled trial & $\begin{array}{l}\text { Observational study design; pilot studies, when a paper clearly } \\
\text { stated that the research was a 'pilot study' }\end{array}$ \\
\hline Intervention & $\begin{array}{l}\text { Dietary intervention either diet alone (a dietary pattern or } \\
\text { dietary supplements) or in combination with lifestyle and/or } \\
\text { cognitive strategies }\end{array}$ & $\begin{array}{l}\text { Medical type intervention in conjunction with either a diet/lifestyle/ } \\
\text { cognitive intervention with undifferentiated results }\end{array}$ \\
\hline Control & $\begin{array}{l}\text { Control interventions that were not expected to have specific } \\
\text { risk-modifying effects; control arms would typically involve } \\
\text { no intervention, usual diet or placebo }\end{array}$ & Studies with no comparator, placebo or control \\
\hline Diagnosis of $\mathrm{MCl}$ & $\begin{array}{l}\text { Diagnosis of } \mathrm{MCl} \text { was necessary by a medical physician or } \\
\text { according to internationally accepted and validated } \\
\text { classifications or criteria }\end{array}$ & $\begin{array}{l}\text { 'Memory problems' or 'self-reported memory complaints' and no } \\
\text { clear diagnosis of } \mathrm{MCl} \text {; a diagnosis of dementia or any other form } \\
\text { of cognitive impairment other than } \mathrm{MCl} \text {, unless results for } \mathrm{MCl} \\
\text { participants were presented separately; 'cognitively healthy adults' }\end{array}$ \\
\hline Participants & $\begin{array}{l}\text { Community dwelling participants; no restrictions made } \\
\text { based on sex or age }\end{array}$ & $\begin{array}{l}\text { Individuals who were hospitalised, in a rehabilitation or long-term care } \\
\text { facility; participants with psychiatric problems, for example, } \\
\text { depression or any significant medical comorbidity, or history of, a } \\
\text { comorbid condition that may alter performance on cognitive tests, } \\
\text { for example, stroke, head injury, Parkinson's disease and learning } \\
\text { disability }\end{array}$ \\
\hline
\end{tabular}

$\mathrm{MCl}$, mild cognitive impairment. 
discussed among the research team (C. T. M., J. V. W., B. M. and M. C. M.). A data extraction form was generated to summarise the key characteristics of the included articles, extracting information on participant, intervention, and methodological characteristics and cognitive outcome results. Data was extracted for the primary and secondary outcomes as stated previously. Information on quality of life and number of participants experiencing one or more serious adverse events was also extracted when provided in papers in addition to the primary and secondary outcomes mentioned. Where studies included validated biomarkers (e.g. structural MRI or amyloid imaging) secondary to cognitive outcome measures, these data were also extracted. The extraction was undertaken by the first author (A. M. M.) and this was independently checked by the second author (C. T. M.) and both reviewers discussed any discrepancies as required.

\section{Quality assessment}

The methodological quality of the included studies was assessed using the Jadad scale ${ }^{(22)}$. This scale has been widely used to assess the quality of RCT included in systematic reviews with regards to randomisation procedures, double blinding and participant withdrawals. A score of 1 was allocated for each 'yes' answer to the following three questions:

(1) Was the study described as randomised?

(2) Was the study described as double blind?

(3) Was there a description of withdrawals and drop outs?

An additional score of 1 was awarded if;
(4) The randomisation process was described and appropriate

(5) The method of double blinding was described and appropriate. The maximum possible score was $5^{(22)}$.

The risk of bias was assessed using the Cochrane classification ${ }^{(23)}$. Each study was assessed for the following (where appropriate): (1) selection bias; (2) performance bias; (3) detection bias; (4) attrition bias and (5) reporting bias. Individual studies were assessed as either low, high or uncertain risk for the adequacy of the stated variables.

\section{Data analysis}

The data collected were expected to display a high degree of heterogeneity, therefore quantitative synthesis was unsuitable. The results were summarised using narrative synthesis and presented in tables.

\section{Results}

The systematic search in June 2016 generated a total of 2130 articles (2108 through database searches and twenty-two through searches of reference lists). Following the removal of 650 duplicates, 1480 articles were screened for eligibility by examining their titles and abstracts. This process excluded 1447 studies and the full texts of thirty-three papers were obtained; twenty-two articles were excluded for the reasons outlined in Fig. 1. Following a second (November 2016) and third (March 2018) literature search, five further studies were identified that met the inclusion criteria and so sixteen studies were included. As per the review protocol, the results have been

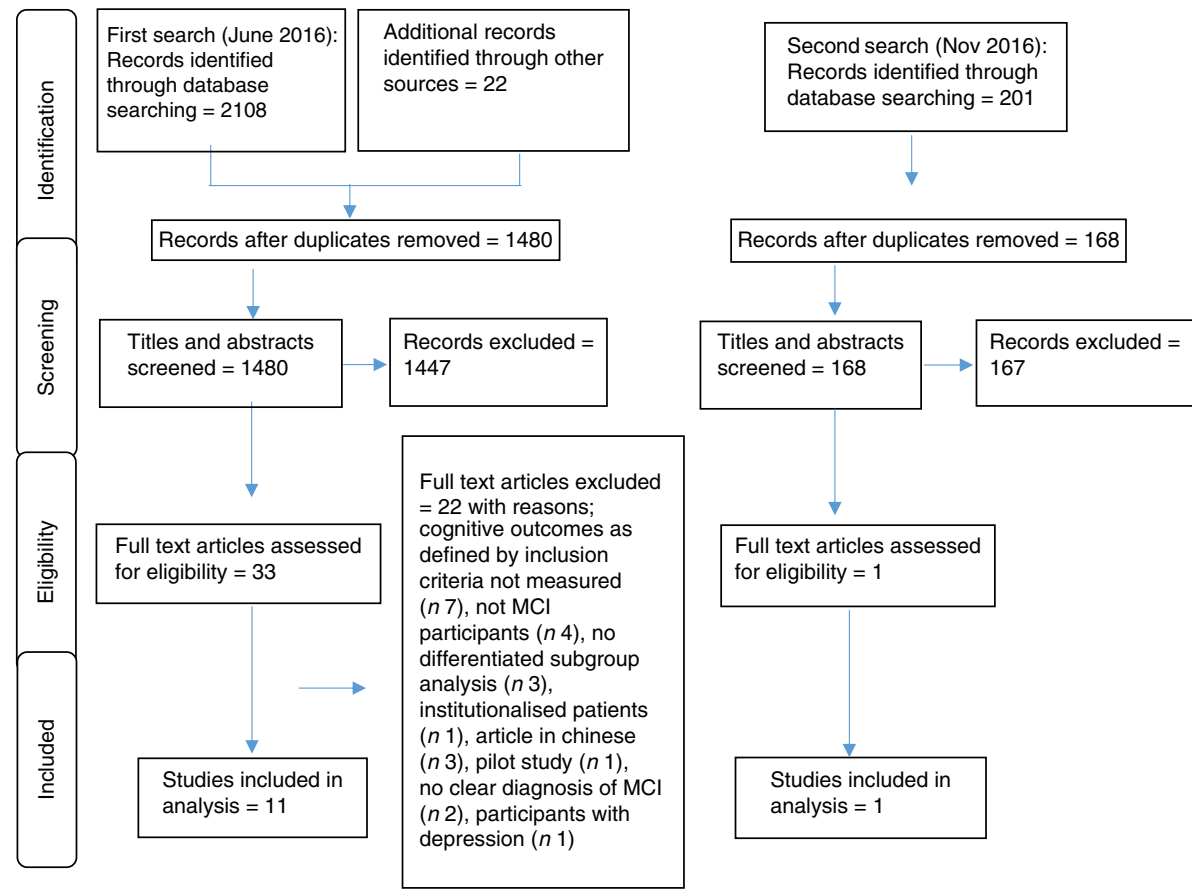

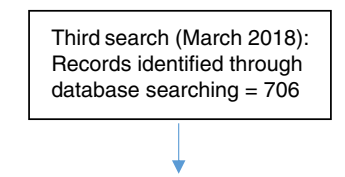

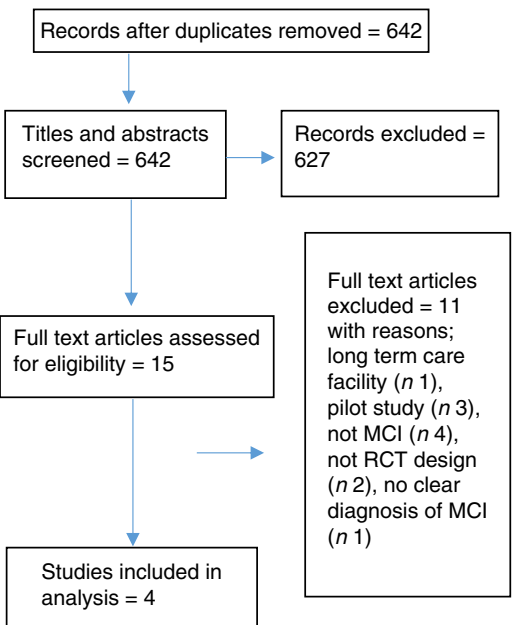

Fig. 1. Preferred Reporting Items for Systematic Reviews and Meta-Analyses (PRISMA) flow diagram. MCl, mild cognitive impairment. 


\section{N British Journal of Nutrition}

Table 2. Overview of study characteristics

\begin{tabular}{|c|c|c|c|c|c|}
\hline $\begin{array}{l}\text { Author, year } \\
\text { and location }\end{array}$ & $\begin{array}{l}\mathrm{MCl} \text { sample } \\
\text { characteristics }\end{array}$ & $\begin{array}{l}\text { Diagnostic criteria } \\
\text { for } \mathrm{MCl}\end{array}$ & Intervention & $\begin{array}{l}\text { Study } \\
\text { duration }\end{array}$ & Outcome measures \\
\hline $\begin{array}{l}\text { Bayer-Carter et al. } \\
(2011)^{(38)}(n \text { 49) } \\
\text { USA }\end{array}$ & $\begin{array}{l}68 \cdot 4 \text { years } \\
\text { aMCl }=29 \\
\text { High }=15 \\
\text { Low }=14 \\
\text { Healthy controls }=20 \\
\text { High }=9 \\
\text { Low }=11 \\
\text { Lost to follow up=no detail }\end{array}$ & Petersen $(2004)^{(82)}$ & $\begin{array}{l}\text { Intervention groups: } \\
\text { (1) High diet }-45 \% \text { fat (saturated fat } 25 \% \text { ), } \\
35-40 \% \text { carbohydrate }(\mathrm{Gl}>70) \text { and } 15-20 \% \\
\text { protein } \\
\text { (2) Low diet }-25 \% \text { fat (saturated fat }<7 \%) \text {, } \\
55-60 \% \text { carbohydrate }(\mathrm{Gl}<55 \text { ) and } 15-20 \% \\
\text { protein } \\
\text { Control group: healthy adult control group }\end{array}$ & 4 weeks & $\begin{array}{l}\text { Immediate and delayed memory: story recall, word } \\
\text { list, brief visuospatial memory test; executive } \\
\text { function: trail making test part B, Stroop test, } \\
\text { verbal fluency test; } \\
\text { motor speed: trail making test part A, Stroop test; } \\
\text { AD biomarkers } \\
\text { CSF A } \beta 42, \text { CSF A } \beta 40 \text {, tau protein } \\
\text { phosphorylated tau, ApoE }\end{array}$ \\
\hline $\begin{array}{l}\text { Horie et al. (2016) } \\
\qquad(n 80)^{(37)} \text { Brazil }\end{array}$ & $\begin{array}{l}68 \cdot 1 \text { years } \\
\text { Intervention }=40 \\
\text { Control }=40 \\
\text { Lost to follow up }=5\end{array}$ & $\begin{array}{l}\text { European } \\
\text { Consortium on } \\
\text { Alzheimer's } \\
\text { Disease }^{(80)}\end{array}$ & $\begin{array}{l}\text { Intervention group: energetic restriction and } \\
\text { counselling with nutritionists }(26-28 \times 1 \mathrm{~h} \\
\text { meetings). Advice-eating a diet rich in fibre, } \\
\text { fruits, vegetables, wholegrains and included at } \\
\text { least } 1 \mathrm{~g} / \mathrm{kg} \text { body weight of protein/d. } \\
\text { Recommended energy deficit of approximately } \\
500 \mathrm{kcal} / \mathrm{d}(2092 \mathrm{~kJ} / \mathrm{d})(\mathrm{min} 1200 \mathrm{kcal} / \mathrm{d}(5021 \mathrm{~kJ} / \mathrm{d})) \\
\text { Control group: conventional medical care with } \\
\text { consultant geriatrician } \\
\text { All participants were advised to engage in physical } \\
\text { activity (at least } 150 \mathrm{~min} / \mathrm{week} \text { of moderate intensity } \\
\text { aerobic activity or walking) }\end{array}$ & 12 months & $\begin{array}{l}\text { Verbal memory: RAVLT delayed recall, total } \\
\text { learning and recall recognition; attention: digit } \\
\text { span forward, digit span backward, trail making } \\
\text { test part A; working memory: digit span } \\
\text { backwards, trail making test part; psychomotor } \\
\text { processing speed: trail making test part A, trail } \\
\text { making test part B; executive function: modified } \\
\text { Wisconsin Card Sorting Test, trail making test } \\
\text { part B, verbal fluency; language: phonemic } \\
\text { verbal fluency, semantic verbal fluency }\end{array}$ \\
\hline $\begin{array}{l}\text { Krikorian et al. } \\
(2012)^{(12)}(n \text { 23) } \\
\quad \text { USA }\end{array}$ & $\begin{array}{l}70 \cdot 1 \text { years } \\
\text { High carbohydrate }=11 \\
\text { Low carbohydrate }=12 \\
\text { Lost to follow up }=0\end{array}$ & $\mathrm{CDR}^{(81)}$ & $\begin{array}{l}\text { Intervention groups: high carbohydrate }(50 \% \text { of } \\
\text { total energy content) } v \text {. a very low carbohydrate } \\
\text { group (5-10\% total energy content). Intake of } \\
\text { protein and fat were allowed to vary and total } \\
\text { energy intake was not restricted }\end{array}$ & 6 weeks & $\begin{array}{l}\text { Working memory and executive ability: trail making } \\
\text { test, part B. Secondary or long term memory: V- } \\
\text { PAL test }\end{array}$ \\
\hline $\begin{array}{l}\text { de Jager et al. } \\
(2012)^{(25)}(n \text { 266) } \\
\text { UK }\end{array}$ & $\begin{array}{l}76 \cdot 8 \text { years } \\
\text { Intervention }=133 \\
\text { Control }=133 \\
\text { Lost to follow up }=43\end{array}$ & $\begin{array}{l}\text { Petersen criteria } \\
(2004)^{(82)}\end{array}$ & $\begin{array}{l}\text { Intervention group: } 0.8 \mathrm{mg} \text { folic acid, } 0.5 \mathrm{mg} \text { vitamin } \\
\mathrm{B}_{12}, 20 \mathrm{mg} \text { vitamin } \mathrm{B}_{6} \text { (daily) } \\
\text { Control group: vitamin-free tablets of similar } \\
\text { appearance }\end{array}$ & 2 years & $\begin{array}{l}\text { Global cognition: MMSE; episodic memory: HVLT- } \\
\text { R; semantic memory: category fluency CERAD; } \\
\text { executive function: CLOX; clinical outcome } \\
\text { measures: CDR }\end{array}$ \\
\hline $\begin{array}{l}\text { Ma et al. }(2016)^{(24)} \\
(n \text { 180) China }\end{array}$ & $\begin{array}{l}65 \text { years } \\
\text { Intervention }=90 \\
\text { Control }=90 \\
\text { Lost to follow up }=21\end{array}$ & $\begin{array}{l}\text { Petersen criteria } \\
(2004)^{(82)}\end{array}$ & $\begin{array}{l}\text { Intervention group: oral folic acid }(400 \mu \mathrm{g} / \mathrm{d}) \text {. } \\
\text { Participants were instructed to supplement with } \\
\text { one tablet daily, during or immediately after a } \\
\text { meal } \\
\text { Control group: conventional medical treatment }\end{array}$ & 6 months & $\begin{array}{l}\text { Chinese version of the WAIS-RC-information, } \\
\text { similarities, vocabulary, comprehension, } \\
\text { arithmetic, digit span, block design, picture } \\
\text { completion, digit symbol-coding, object assembly } \\
\text { and picture arrangement }\end{array}$ \\
\hline $\begin{array}{l}\text { DeKosky et al. } \\
(2008)^{(26)} \text { (total } \\
\text { study } n 3069, n \\
482 \text { with MCl) USA }\end{array}$ & $\begin{array}{l}79 \cdot 1 \text { years } \\
\text { Intervention=256 } \\
\text { Control=226 } \\
\text { Lost to follow up: } 195 \text { (total } \\
\text { study) }\end{array}$ & $\begin{array}{l}\text { International Working } \\
\text { Group on } \mathrm{MCl} \\
\text { Guidelines }{ }^{(83)}\end{array}$ & $\begin{array}{l}\text { Intervention group: twice-daily doses of } 120 \mathrm{mg} \\
\text { G. biloba extract } \\
\text { Control group: received an identically appearing } \\
\text { placebo }\end{array}$ & 6.1 years & $\begin{array}{l}\text { Diagnosis of dementia by DSM-IV criteria, modified } \\
\text { MMSE, CDR, ADAS-Cog }\end{array}$ \\
\hline $\begin{array}{l}\text { Lee et al. }(2013)(n \\
36)^{(27)} \text { Malaysia }\end{array}$ & $\begin{array}{l}65 \cdot 0 \text { years } \\
\text { Intervention }=18 \\
\text { Control }=18 \\
\text { Lost to follow up }=1\end{array}$ & $\begin{array}{l}\text { Petersen criteria } \\
(2004)^{(82)}\end{array}$ & $\begin{array}{l}\text { Intervention group: } 3 \times 1-\mathrm{g} \text { soft gelatine capsules } \\
\text { each day, each containing } 430 \mathrm{mg} \text { of DHA and } \\
150 \mathrm{mg} \text { of EPA } \\
\text { Control group: isoenergetic placebo maize oil }(0.6 \mathrm{~g} \\
\text { linoleic acid) }\end{array}$ & 12 months & $\begin{array}{l}\text { Memory: visual reproduction I and II, RAVLT, digit } \\
\text { span backward; executive function and attention: } \\
\text { clock drawing test, digit span forward; } \\
\text { psychomotor speed: digit symbol substitution } \\
\text { test; visuospatial skills: matrix reasoning, block } \\
\text { design; global cognitive function: MMSE }\end{array}$ \\
\hline
\end{tabular}




\section{N British Journal of Nutrition}

Table 2. Continued

\begin{tabular}{|c|c|c|c|c|c|}
\hline $\begin{array}{l}\text { Author, year } \\
\text { and location }\end{array}$ & $\begin{array}{l}\mathrm{MCl} \text { sample } \\
\text { characteristics }\end{array}$ & $\begin{array}{l}\text { Diagnostic criteria } \\
\text { for } \mathrm{MCl}\end{array}$ & Intervention & $\begin{array}{l}\text { Study } \\
\text { duration }\end{array}$ & Outcome measures \\
\hline $\begin{array}{l}\text { Petersen et al. } \\
(2005)^{(31)} \text { (total } \\
\text { study } n \text { 769) USA } \\
\text { and Canada }\end{array}$ & $\begin{array}{l}72 \cdot 9 \text { years } \\
\text { Donepezil }=253 \\
\text { Vitamin } E=257 \\
\text { Placebo }=259 \\
\text { Lost to follow up: } 230 \text { (total } \\
\text { study) }\end{array}$ & Petersen $(1999)^{(84)}$ & $\begin{array}{l}\text { Intervention group: } \\
\text { (1) } 2000 \text { IU ( } 1.3 \mathrm{mg} \text { ) of vitamin E, placebo } \\
\text { donepezil and a multivitamin daily; (2) } 10 \mathrm{mg} \text { of } \\
\text { donepezil, placebo vitamin E and a multivitamin } \\
\text { daily. The multivitamin contained } 15 \mathrm{IU} \text { of vitamin } \\
\text { E. The initial dose of vitamin E was } 1000 \mathrm{IU} \text { daily, } \\
\text { and the dose was increased to } 2000 \mathrm{IU} \text { ( } 1000 \mathrm{IU} \\
\text { twice daily) after } 6 \text { weeks } \\
\text { Control group: received a placebo vitamin E, } \\
\text { placebo donepezil and a multivitamin daily }\end{array}$ & 3 years & $\begin{array}{l}\text { Primary end-point: time to development of possible } \\
\text { of probable Alzheimer's disease; secondary: } \\
\text { MMSE, ADAS-Cog, global CDR, } \\
\text { CDR-SB, the global deterioration scale } \\
\text { neuropsychological battery consisting of: New } \\
\text { York University paragraph recall test, symbol } \\
\text { digit modalities test, category fluency test, } \\
\text { number-cancellation test, Boston naming test, } \\
\text { digits-backwards test, clock drawing test, maze } \\
\text { tracing task }\end{array}$ \\
\hline $\begin{array}{l}\text { Desideri et al. } \\
\quad(2012)^{(34)}(n 90) \\
\text { Italy }\end{array}$ & $\begin{array}{l}71 \cdot 2 \text { years } \\
\text { High }=30 \\
\text { Medium }=30 \\
\text { Low }=30 \\
\text { Lost to follow up: } 3 \\
\quad \text { (included in analysis) }\end{array}$ & $\begin{array}{l}\text { Petersen criteria } \\
(2004)^{(82)}\end{array}$ & $\begin{array}{l}\text { Intervention group: once daily a dairy-based cocoa } \\
\text { drink containing cocoa flavanols either at - (1) } \\
\text { high flavanol ( } 990 \mathrm{mg} \text { of flavanols per serving), } \\
\text { (2) intermediate flavanol ( } 520 \mathrm{mg} \text { of flavanols per } \\
\text { serving) and (3) low flavanol ( } 45 \mathrm{mg} \text { of flavanols } \\
\text { per serving) }\end{array}$ & 8 weeks & $\begin{array}{l}\text { MMSE, trail making test A and B, verbal fluency } \\
\text { test }\end{array}$ \\
\hline $\begin{array}{l}\text { Krikorian et al. } \\
(2010)^{(35)}(n 12) \\
\text { USA }\end{array}$ & $\begin{array}{l}72 \cdot 8 \text { years } \\
\text { Male and female } \\
\text { Intervention }=5 \\
\text { Control }=7 \\
\text { Lost to follow up }=0\end{array}$ & $\mathrm{CDR}^{(81)}$ & $\begin{array}{l}\text { Intervention group: } 100 \% \text { concord grape juice. A } \\
\text { dosing schedule was instituted determined by } \\
\text { body weight to maintain daily consumption } \\
\text { between } 6 \text { and } 9 \mathrm{ml} / \mathrm{kg} \text {, a range consistent with } \\
\text { other human grape juice trials. Taken daily in } \\
\text { equal, divided dosages with the morning, midday } \\
\text { and evening meals } \\
\text { Control: contained no juice or natural polyphenol }\end{array}$ & 12 weeks & $\begin{array}{l}\text { Memory: CVLT, spatial paired associate } \\
\text { learning test }\end{array}$ \\
\hline $\begin{array}{l}\text { Krikorian et al. } \\
\quad(2010)^{(36)}(n \text { 9) } \\
\text { USA }\end{array}$ & $\begin{array}{l}76 \cdot 2 \text { years } \\
\text { Male and female } \\
\text { Intervention }=\text { no detail } \\
\text { Control=no detail } \\
\text { Lost to follow up }=\text { no detail }\end{array}$ & $\mathrm{CDR}^{(81)}$ & $\begin{array}{l}\text { Intervention group: wild blueberry juice prepared } \\
\text { from ripe, frozen wild (lowbush) blueberries. } \\
\text { Taken daily in equal divided dosages with } \\
\text { morning, mid-day and evening meals. Daily } \\
\text { consumption was maintained between } 6 \text { and } 9 \\
\text { ml/kg by using a dosing schedule determined by } \\
\text { body weight } \\
\text { Control: contained no juice or natural polyphenol }\end{array}$ & 12 weeks & Memory: V-PAL test and CVLT \\
\hline $\begin{array}{l}\text { Krikorian et al. } \\
(2010)^{(32)}(n \text { 26) } \\
\text { USA }\end{array}$ & $\begin{array}{l}71.0 \text { years } \\
\text { Intervention }=15 \\
\text { Control }=11 \\
\text { Lost to follow up=no detail }\end{array}$ & $\mathrm{CDR}^{(81)}$ & $\begin{array}{l}\text { Intervention group: chromium picolinate containing } \\
1000 \mu \mathrm{g} \text { elemental } \mathrm{Cr} \\
\text { Control group: placebo - no details }\end{array}$ & 12 weeks & $\begin{array}{l}\text { Memory: CVLT } \\
\quad \text { functional MRI scanning }\end{array}$ \\
\hline $\begin{array}{l}\text { Bo et al. (2017) }(n \\
\text { 86) } \\
\text { (28) China }\end{array}$ & $\begin{array}{l}71.1 \text { years } \\
\text { Intervention }=42 \\
\text { Control }=44 \\
\text { Lost to follow up }=22\end{array}$ & $\begin{array}{l}\text { Petersen criteria } \\
\quad(1999)^{(84)}\end{array}$ & $\begin{array}{l}\text { Intervention group: } 625 \text { mg DHA+600 mg EPA } \\
\text { (twice daily) } \\
\text { Control group: placebo capsules containing olive oil } \\
\text { (twice daily) }\end{array}$ & 6 months & $\begin{array}{l}\text { Basic cognitive aptitude tests: digit copy, Chinese } \\
\text { character comparison, mental arithmetic, } \\
\text { Chinese character rotation, recall answer of } \\
\text { mental arithmetic, recognition of two-word } \\
\text { nouns, and recognition of meaningless figures. } \\
\text { These seven sub-items were divided into five } \\
\text { sections: perceptual speed, mental arithmetic } \\
\text { efficiency, space imagery efficiency, working } \\
\text { memory and recognition memory }\end{array}$ \\
\hline
\end{tabular}




\section{N British Journal of Nutrition}

Table 2. Continued

\begin{tabular}{|c|c|c|c|c|c|}
\hline $\begin{array}{l}\text { Author, year } \\
\text { and location }\end{array}$ & $\begin{array}{l}\mathrm{MCl} \text { sample } \\
\text { characteristics }\end{array}$ & $\begin{array}{l}\text { Diagnostic criteria } \\
\text { for } \mathrm{MCl}\end{array}$ & Intervention & $\begin{array}{l}\text { Study } \\
\text { duration }\end{array}$ & Outcome measures \\
\hline $\begin{array}{l}\text { Soininen et al. } \\
(2017)^{(33)}(n \text { 311) } \\
\text { Finland }\end{array}$ & $\begin{array}{l}71.0 \text { years } \\
\text { Intervention }=153 \\
\text { Control=158 } \\
\text { Lost to follow up/ } \\
\quad \text { discontinued }=66\end{array}$ & $\begin{array}{l}\text { Dubois et al. } \\
\qquad(2007)^{(85)}\end{array}$ & $\begin{array}{l}\text { Intervention group: medical food Souvenaid, a } 125 \\
\text { ml once-a-day drink containing the specific } \\
\text { nutrient combination Fortasyn Connect }(1200 \mathrm{mg} \\
\text { DHA, } 300 \mathrm{mg} \text { EPA, } 106 \mathrm{mg} \text { phospholipids, } 400 \\
\text { mg choline, } 625 \mathrm{mg} \text { UMP, } 40 \mathrm{mg} \text { vitamin E, } 80 \\
\text { mg vitamin } \mathrm{C}, 60 \mu \mathrm{g} \mathrm{Se}, 3 \mu \mathrm{g} \text { vitamin } \mathrm{B}_{12}, 1 \mathrm{mg} \\
\text { vitamin } \mathrm{B}_{6}, 400 \mu \mathrm{g} \text { folic acid) } \\
\text { Control group: } 125 \mathrm{ml} \text { once-a-day control drink }\end{array}$ & 24 months & $\begin{array}{l}\text { Primary end points: composite } Z \text { score based on } \\
\text { CERAD ten-word list learning immediate recall, } \\
\text { CERAD ten-word delayed recall, CERAD ten- } \\
\text { word recognition, category fluency and LDST. } \\
\text { Memory (CERAD ten-word list learning } \\
\text { immediate recall, delayed recall and recognition); } \\
\text { executive function (category fluency, Wechsler } \\
\text { Memory Scale revised digit span total score, } \\
\text { concept shifting test condition C (corrected for } \\
\text { the zero trials) and LDST); NTB total (composite } \\
Z \text { score based on all sixteen items of the NTB); } \\
\text { secondary end points: CDR-SB; brain volumes } \\
\text { based on MRI; progression to dementia by DSM- } \\
\text { IV Criteria }\end{array}$ \\
\hline $\begin{array}{l}\text { Zhang et al. (2017) ( } \\
\text { 240) } \\
{ }^{(30)} \text { China }\end{array}$ & $\begin{array}{l}74.5 \text { years } \\
\text { Intervention }=120 \\
\text { Control }=120 \\
\text { Lost to follow up }=21\end{array}$ & $\begin{array}{l}\text { Petersen criteria } \\
\quad(2004)^{(82)}\end{array}$ & $\begin{array}{l}\text { Intervention group: DHA supplementation }(2 \mathrm{~g} / \mathrm{d}) \\
\text { Control group: maize oil }\end{array}$ & 12 months & $\begin{array}{l}\text { Chinese version of the WAIS-RC. The WAIS-RC } \\
\text { includes eleven sub-tests: information, } \\
\text { similarities, vocabulary, comprehension, } \\
\text { arithmetic, digit span, block design, picture } \\
\text { completion, digit symbol-coding, object assembly } \\
\text { and picture arrangement }\end{array}$ \\
\hline $\begin{array}{l}\text { Phillips et al. } \\
\quad(2015)^{(29)}(n 57) \\
\text { UK }\end{array}$ & $\begin{array}{l}68 \cdot 7 \text { years } \\
\text { Intervention }=29 \\
\text { Control }=28 \\
\text { Lost to follow up }=2\end{array}$ & $\begin{array}{l}\text { Petersen criteria } \\
\quad(2004)^{(82)}\end{array}$ & $\begin{array}{l}\text { Intervention group: } 625 \mathrm{mg} \text { DHA+600 mg EPA } \\
\text { (twice daily) } \\
\text { Control group: placebo capsules containing olive oil } \\
\text { (twice daily) }\end{array}$ & 4 months & $\begin{array}{l}\text { MMSE; Hopkins Learning Test Revised and } \\
\text { neuropsychological measures of executive } \\
\text { functioning, language, verbal reasoning and } \\
\text { visual memory }\end{array}$ \\
\hline
\end{tabular}


displayed according to the primary (incident dementia or AD) and secondary (cognitive function) outcomes. For cognitive function, as per the NIA-AA criteria for the diagnosis of $\mathrm{MCI}^{(3)}$, the results were grouped according to the following cognitive domains: (1) memory; (2) executive function; (3) attention; (4) language and (5) visuospatial skills, with an additional section reporting global cognitive function. When papers did not specify the cognitive domain measured, the results were grouped under 'additional cognitive function measures' (online Supplementary Table S2). A descriptive list of the most frequently reported cognitive function tests used in the studies is provided in the online Supplementary material.

\section{Study characteristics}

An overview of the study characteristics is shown in Table 2. Of the sixteen studies included in analysis, thirteen studies used dietary supplements or single foods as their diet intervention, including folic acid ${ }^{(24)}$, vitamin $\mathrm{B}$ combination (folic acid, vitamin $\mathrm{B}_{12}$ and vitamin $\left.\mathrm{B}_{6}\right)^{(25)}$, Gingko biloba ${ }^{(26)}, n-3$ fatty acids (DHA $+\mathrm{EPA}^{(27-29)}$ and $\left.\mathrm{DHA}^{(30)}\right)$, vitamin $\mathrm{E}^{(31)}$, Cr supplementation ${ }^{(32)}$, the medical food, Souvenaid containing the specific nutrition combination Fortasyn Connect ${ }^{(33)}$, cocoa flavanols ${ }^{(34)}$, concord grape juice ${ }^{(35)}$ and wild blueberry juice ${ }^{(36)}$. The three remaining studies focused their interventions on nutritional counselling in combination with healthy eating advice and energy restriction ${ }^{(37)}$, high-saturated fat/ high-glycaemic index (GI) diet $v$. a low-saturated fat/low-GI diet ${ }^{(38)}$ and a high carbohydrate $v$. a very low carbohydrate diet ${ }^{(12)}$. A figure detailing the included studies and their dietary exposure linked to the cognitive outcome measures assessed is provided in the online Supplementary material (Fig. 1). One study ${ }^{(37)}$ encouraged both intervention and control participants to partake in physical activity ( $150 \mathrm{~min} /$ week) as per World Health Organization ${ }^{(39)}$ recommendations. There were no studies which included cognitive strategies as part of their intervention. Furthermore, two studies stated that participants had amnesic mild cognitive impairment (aMCI) ${ }^{(38)}$ or prodromal $\mathrm{AD}^{(33)}$ while all other studies reported a diagnosis of MCI.

\section{Primary outcome measure - incident dementia and Alzheimer's disease}

In all, three of the included studies had an outcome measure of incident dementia and/or $\mathrm{AD}^{(26,31,33)}$. Vitamin E supplementation over 3 years showed no significant difference in the diagnostic rate of $\mathrm{AD}$ in participants with MCI taking vitamin $\mathrm{E}$ (2000 IU) $v$. placebo (hazard ratio (HR) $1.02,95 \%$ CI $0.57,1 \cdot 13)^{(31)}$. In the vitamin E group, 33/257 (13\%) and 38/259 (15\%) participants in the placebo group progressed to possible or probable $\mathrm{AD}$ in the first 12 months (relative risk (RR) 1.02, $95 \%$ CI 0.96, 1.10). At 36 months, $76 / 257$ (30\%) in the vitamin E group and 73/259 (28\%) in the placebo had progressed to AD (RR $1 \cdot 03,95 \% \mathrm{CI}$ $0.79,1.35)^{(31)}$. Likewise, a USA based study with intervention follow up over $6 \cdot 1$ years and found no significant difference between $G$. biloba $v$. placebo for the outcomes of all dementia (9.82/100 person-years $v$. 8.68/100 person-years, HR $1 \cdot 13,95 \%$ CI $0 \cdot 85,1 \cdot 50)$, AD without vascular dementia (VaD) $(7 \cdot 02 / 100$ person-years $v$. 6.09/100 person-years, HR $1 \cdot 15$, 95\% CI $0 \cdot 83$, 1.61), AD with $\operatorname{VaD}(2 \cdot 10 / 100$ person-years $v \cdot 2 \cdot 20 / 100$ person years, HR $0 \cdot 96,95 \%$ CI $0 \cdot 54,1 \cdot 71)$, total AD $(9 \cdot 12 / 100$ personyears $v .8 \cdot 28 / 100$ person-years, HR $1 \cdot 10,95 \%$ CI $0 \cdot 83,1 \cdot 47)$ and $\mathrm{VaD}$ without $\mathrm{AD}(0 \cdot 18 / 100$ person-years $v \cdot 0 \cdot 30 / 100$ personyears, HR $0.59,95 \%$ CI $0 \cdot 10,3.51)^{(26)}$. Finally, supplementation with Souvenaid $(125 \mathrm{ml} / \mathrm{d}$ of the specific nutrition combination Fortasyn Connect) $v$. control, showed no statistically significant difference in diagnosis of dementia at 24 months between groups (59/158 (37\%) (control) v. 62/153 (41\%) (intervention) $)^{(33)}$.

\section{Secondary outcome measure-cognitive function}

Memory. As shown in Table 3, there were twenty-five cognitive tests used to measure the domain of memory, and it was assessed in fifteen out of the sixteen studies (94\%) and hence was the most tested cognitive domain. Overall, nine out of the fifteen studies (53\%) (B vitamin ${ }^{(25)}, \mathrm{DHA}+\mathrm{EPA}^{(27-29)}, \mathrm{DHA}^{(30)}$, vitamin $\mathrm{E}^{(31)}$, cocoa flavonols ${ }^{(34)}$, concord grape juice ${ }^{(35)}$ and wild blueberry juice ${ }^{(36)}$ ) showed a significant difference between groups at study completion in at least one cognitive function test measuring memory. Fish oil supplementation $(3 \times 430 \mathrm{mg}$ DHA $+150 \mathrm{mg}$ EPA daily for 12 months), produced significant improvements in visual reproduction I and Rey Auditory Verbal Learning Test delayed recall $v$. placebo group (all $P<0.05)^{(27)}$. In addition, there was a significant improvement in memory performance (cognitive $Z$ score) in the fish oil $v$. placebo group $(P=0 \cdot 001)^{(27)}$. In a second study investigating $n$-3 PUFA supplementation ( $480 \mathrm{mg}$ DHA $+720 \mathrm{mg}$ EPA daily for 6 months $v$. placebo $)^{(28)}$, borderline statistical significance $(P=0.047)$ was reported between intervention and control for working memory. However, a third study investigating $625 \mathrm{mg} \mathrm{EPA}+600 \mathrm{mg}$ DHA $v$. placebo showed no significant improvements in memory ${ }^{(29)}$. A fourth study who investigated DHA supplementation only $(2 \mathrm{mg} / \mathrm{d} v \text {. placebo })^{(30)}$, found significant improvements for short-term memory $(P \leq 0.0001)$ and long-term memory $(P \leq 0.0001)$ in comparison to the placebo group. In a trial investigating the effect of cocoa flavanols (high flavonols (HF) $990 \mathrm{mg} v$. intermediate flavonols (IF) $520 \mathrm{mg} v$. low flavonols (LF) $45 \mathrm{mg}$ of flavanols daily for 8 weeks) ${ }^{(34)}$, verbal fluency test scores significantly improved ( $P=0 \cdot 0001)$, with a significantly greater score in HF participants in comparison with the LF group $(P \leq 0.05)$.

$\mathrm{B}$ vitamin supplementation ${ }^{(25)}(0.8 \mathrm{mg}$ folic acid, $0.5 \mathrm{mg}$ vitamin $\mathrm{B}_{12}, 20 \mathrm{mg}$ vitamin $\mathrm{B}_{6}$ daily for 2 years), demonstrated improvement in verbal memory but only in those participants with low baseline B vitamin/folic acid status. The odds of correctly remembering a word in the Hopkins Verbal Learning Test were $69 \%$ greater for a person in the high total homocysteine (tHcy) group if they were taking B vitamins, than if they were taking placebo $(\mathrm{OR}=1 \cdot 69, P=0 \cdot 001)^{(25)}$. For category fluency (Consortium to Establish a Registry for Alzheimer's Disease), in the high tHcy group, the average number of words was $9.4 \%$ greater at follow up in those on $\mathrm{B}$ vitamin treatment compared with the placebo $(P=0 \cdot 04)$. However, in the low tHcy group (indicating higher B vitamin/ folic acid status) there was no significant difference between the 
Table 3. Summary table of cognitive function results grouped as per National Institute for Aging-Alzheimer's Association (NIA-AA) ${ }^{(3)}$ criteria

\begin{tabular}{|c|c|c|c|c|c|}
\hline $\begin{array}{l}\text { NIA-AA cognitive } \\
\text { domain }\end{array}$ & Study & Intervention & Cognitive function measure used & Intervention group and control group results & $\begin{array}{l}\text { Between group } \\
\text { difference }\end{array}$ \\
\hline \multirow[t]{15}{*}{ Memory } & \multirow[t]{4}{*}{ Horie et al., 2016 } & \multirow[t]{4}{*}{$\begin{array}{l}\text { Nutrition counselling and energy } \\
\text { restriction } v \text {. standard care }\end{array}$} & RAVLT (delayed recall) & $\begin{array}{l}\text { Intervention (mean change } 0.7,95 \% \mathrm{Cl}-0.9,2 \cdot 3 \text { ); control } \\
\text { (mean change } 1.7,95 \% \mathrm{Cl} 0.1,3 \cdot 3 \text { ) }\end{array}$ & $\ddagger$ \\
\hline & & & RAVLT (total learning) & $\begin{array}{l}\text { Intervention (mean change } 3 \cdot 3,95 \% \mathrm{Cl}-1 \cdot 3,7 \cdot 9 \text { ); control } \\
\quad \text { (mean change } 2 \cdot 0,95 \% \mathrm{Cl}-2 \cdot 6,6 \cdot 7 \text { ) }\end{array}$ & $\ddagger$ \\
\hline & & & Digit span backward & Intervention $(0.2,95 \% \mathrm{Cl}-0.8,1 \cdot 2)$; control $(0.1,95 \% \mathrm{Cl}-0.9,1 \cdot 1)$ & $\ddagger$ \\
\hline & & & Trail making test, part B & $\begin{array}{l}\text { Intervention (mean change }-8 \cdot 6,95 \% \mathrm{Cl}-71 \cdot 6,54.5) \text {; control } \\
\text { (mean change } 5 \cdot 1,95 \% \mathrm{Cl}-58 \cdot 3,68 \cdot 6 \text { ) }\end{array}$ & $\ddagger$ \\
\hline & \multirow[t]{5}{*}{ Lee et al. $(2013)^{(27)}$} & \multirow[t]{5}{*}{$\begin{array}{l}\text { Fish oil supplementation with } \\
\text { concentrated DHA +EPA } v \text {. } \\
\text { placebo }\end{array}$} & RAVLT (delayed recall) & $\begin{array}{l}\text { Intervention (baseline mean score } 6 \cdot 7,95 \% \mathrm{Cl} 4.897,8.442- \\
12 \text { months mean score } 8.1,95 \% \mathrm{Cl} 6.645,9.462) \text {; control } \\
\text { (baseline mean score } 6 \cdot 1,95 \% \mathrm{Cl} 4.431,7.860-12 \text { months } \\
\text { mean score } 5.0,95 \% \mathrm{Cl} 3.587,6 \cdot 312 \text { ) }\end{array}$ & * \\
\hline & & & Visual reproduction I & $\begin{array}{l}\text { Intervention (baseline mean score } 20 \cdot 0,95 \% \mathrm{Cl} 15 \cdot 234,24 \cdot 820 \text { - } \\
12 \text { months mean score } 29 \cdot 2,95 \% \mathrm{Cl} 25 \cdot 207,33 \cdot 269 \text { ); control } \\
\text { (baseline mean score } 21 \cdot 0,95 \% \mathrm{Cl} 16 \cdot 394,25 \cdot 666 \text { - } 12 \text { months } \\
\text { mean score } 23 \cdot 1,95 \% \mathrm{Cl} 19 \cdot 154,26 \cdot 952 \text { ) }\end{array}$ & * \\
\hline & & & Visual reproduction II & $\begin{array}{l}\text { Intervention (baseline mean score } 13 \cdot 3,95 \% \mathrm{Cl} 8 \cdot 297,18 \cdot 362- \\
12 \text { months mean score } 20 \cdot 8,95 \% \mathrm{Cl} 15 \cdot 564,26 \cdot 110 \text { ); control } \\
\text { (baseline mean score } 12 \cdot 6,95 \% \mathrm{Cl} 7 \cdot 710,17.445-12 \text { months } \\
\text { mean score } 18 \cdot 0,95 \% \mathrm{Cl} 12 \cdot 943,23 \cdot 143 \text { ) }\end{array}$ & $\ddagger$ \\
\hline & & & Digit symbol substitution & $\begin{array}{l}\text { Intervention (baseline mean score } 5.5,95 \% \mathrm{Cl} 3.723,7.218 \text { - } \\
12 \text { months mean score } 5.5,95 \% \mathrm{Cl} 3.723,7 \cdot 218 \text { ); control } \\
\text { (baseline mean score } 4.9,95 \% \mathrm{Cl} 3.254,6.634-12 \text { months } 4.9 \text {, } \\
95 \% \mathrm{Cl} 3.254,6.634 \text { ) }\end{array}$ & $\ddagger$ \\
\hline & & & Memory cognitive $Z$-score & $\begin{array}{l}\left.\text { Intervention (mean change } 0.96(\mathrm{SD} 0.76)^{\star \star \star}\right) \text {; control (mean change } \\
0.16,(\mathrm{SD} 0.59) \text { ) }\end{array}$ & * \\
\hline & $\begin{array}{l}\text { Petersen et al. } \\
\qquad(2005)^{(31)}\end{array}$ & $\begin{array}{l}2000 \mathrm{IU} \text { vitamin } \mathrm{E}, 10 \mathrm{mg} \\
\text { donepezil or placebo }\end{array}$ & $\begin{array}{l}\text { Memory } Z \text { score (ADAS recall scores } \\
\text { and New York University recall } \\
\text { scores) }\end{array}$ & $\begin{array}{l}\text { Intervention ( } 6 \text { months, } Z \text { score }-0.10 \text {, sD } 0.48 ; 36 \text { months } Z \text { score }- \\
\quad 0.31 \text {, sD } 0.59 \text { ); control }(6 \text { months, } Z \text { score }-0.17 \text {, sD } 0.47 \text {; } \\
36 \text { months } Z \text { score }-0.28 \text {, sD } 0.62)\end{array}$ & $\ddagger$ \\
\hline & Ma et al. $(2016)^{(24)}$ & $\begin{array}{l}\text { Oral folic acid }(400 \mu \mathrm{g} / \mathrm{d}) \mathrm{v} \text {. } \\
\text { conventional treatment }\end{array}$ & Digit span & $\begin{array}{l}\text { Intervention (baseline mean score } 9.27 \text { (sD } 3.11)-6 \text { months mean } \\
\text { score 13.05 (sD 3.07); control (baseline mean score } 8.87 \\
\text { (SD 2.70) - } 6 \text { months mean score } 9.75 \text { (SD 3.14) }\end{array}$ & * \\
\hline & \multirow[t]{2}{*}{$\begin{array}{l}\text { De Jager et al. } \\
\qquad(2012)^{(25)}\end{array}$} & \multirow[t]{2}{*}{$\begin{array}{l}0.8 \mathrm{mg} \text { folic acid, } 0.5 \mathrm{mg} \text { vitamin } \\
\mathrm{B}_{12} \text { and } 20 \mathrm{mg} \text { vitamin } \mathrm{B}_{6} \mathrm{~V} \\
\text { placebo }\end{array}$} & $\begin{array}{l}\text { HVLT-R (subgroup analyses, } \\
\text { with baseline tHcy levels) }\end{array}$ & $\begin{array}{l}\text { The odds of correctly remembering a word from the list of twelve in } \\
\text { the HVLT test were } 69 \% \text { greater for a person in the high tHcy } \\
\text { group if they were taking B vitamins than if they were taking } \\
\text { placebo (OR =1.69) }\end{array}$ & * \\
\hline & & & $\begin{array}{l}\text { CERAD (subgroup analyses, } \\
\text { with baseline tHcy levels) }\end{array}$ & $\begin{array}{l}\text { The average number of words was } 9.4 \% \text { greater at follow up in } \\
\text { those on } B \text { vitamin treatment in the high thcy group, compared } \\
\text { with the placebo }(O R=0.09)\end{array}$ & * \\
\hline & \multirow[t]{2}{*}{$\begin{array}{l}\text { Bayer-Carter et al. } \\
\qquad(2011)^{(38)}\end{array}$} & \multirow[t]{2}{*}{$\begin{array}{l}\text { High fat/high GI diet } v \text {. } \\
\text { low fat/low Gl diet }\end{array}$} & Brief visuospatial memory test & $\begin{array}{l}\text { aMCI low diet baseline mean score } 7.39 \text { (SEM } 0.71 \text { ) - week } 4 \text { mean } \\
\text { Score } 8.31 \text { (SEM } 0.62 \text { ); aMCI high diet baseline mean score } 8.27 \\
\text { (SEM } 0.66 \text { ) - week } 4 \text { mean score } 8.40 \text { (SEM } 0.58 \text { ); healthy controls } \\
\text { high diet baseline mean score } 9.89 \text { (SEM } 0.85 \text { ) week } 4 \text { mean } \\
\text { score } 9.56 \text { (SEM } 0.74 \text { ); healthy controls low diet baseline mean } \\
\text { Score of } 8.27 \\
\text { (SEM } 0.77 \text { ) - week } 4 \text { mean score } 9.82 \text { (SEM } 0.67 \text { ) }\end{array}$ & $\ddagger$ \\
\hline & & & Story recall & $\begin{array}{l}\text { aMCI low diet baseline mean score } 18.48 \text { (SEM } 1.43 \text { ) - week } 4 \text { mean } \\
\text { Score 21.46 (SEM } 1.70 \text { ); aMCI high diet baseline mean score } 20.37 \\
\text { (SEM 1.31) - week } 4 \text { mean score 22.30 (SEM } 1.59 \text { ); healthy controls } \\
\text { high diet baseline mean score } 22.69 \text { (SEM } 1.74 \text { ) - week } 4 \text { mean } \\
\text { score } 2319 \text { (SEM 2.04); healthy controls low diet baseline mean } \\
\text { score } 21.09 \text { (SEM 1.55) - week } 4 \text { mean score } 19.90 \text { (SEM 1.95) }\end{array}$ & $\ddagger$ \\
\hline
\end{tabular}


aMCI low diet baseline mean score 11.62 (SEM 0.76) - week 4 mean score 11.77 (SEM 0.80), aMCI high diet baseline mean score 11.33 (SEM 0.71); healthy controls low diet baseline mean score 13.27 (SEM 0.93) - week 4 mean score 13.27 (SEM 0.96), healthy controls high diet baseline mean score 12.79 (SEM 0.92) - week 4 mean score 13.67 (SEM 0.95)

$\begin{array}{cll}\begin{array}{c}\text { Krikorian et al. } \\ (2012)^{(12)}\end{array} & \begin{array}{c}\text { High carbohydrate } v \text {. a very low } \\ \text { carbohydrate }\end{array} & \text { Trail making te } \\ \text { V-PAL } \\ \begin{array}{c}\text { Krikorian et al. } \\ (2010)^{(35)}\end{array} & \begin{array}{c}\text { Concord grape juice } \\ \text { supplementation } v \text {. placebo }\end{array} & \text { CVLT learning }\end{array}$

CVLT recall

Spatial paired associate learning task

$\begin{array}{cc}\text { Krikorian et al. } & \text { Wild blueberry juice } \\ (2010)^{(36)} & \text { supplementation } v \text {. placebo }\end{array}$
$\mathrm{V}-\mathrm{PAL}$

CVLT

Krikorian et al. (2010) ${ }^{(32)}$

Chromium picolinate supplementation $v$. placebo

CVLT learning

\section{CVLT delay recall}

CVLT long delay recall

CVLT recognition memory

$$
\begin{gathered}
\text { Desideri et al. } \\
(2012)^{(34)}
\end{gathered}
$$

$990 \mathrm{mg} \mathrm{HF} v$. IF $v$. LF cocoa flavanols per day

Verbal fluency

Bo et al. $(2017)^{(28)}$ $480 \mathrm{mg}$ of DHA $+720 \mathrm{mg}$ of EPA Working memory daily $v$. placebo

Recognition memory

Soininen et al $(2017)^{(33)}$ Zhang et al.
$(2017)^{(30)}$

Souvenaid, a $125 \mathrm{ml}$ once-a-day drink $v$. control $2 \mathrm{~g} / \mathrm{d}$ DHA v. placebo

NTB memory $Z$ score Information test

Digit span

Immediate verbal memory
Intervention (pre-intervention mean score $79.2 \mathrm{~s} v$ post intervention mean score $82.9 \mathrm{~s}, F_{1,20}=0.46, P=0.50$ ); control (no detail) Intervention (pre-intervention mean score $11.8 \mathrm{~s} v$. post intervention mean score $14.6 \mathrm{~s}, F_{1,20}=6.45, P=0.01$ ); control (no detail) Intervention mean change 3.4; control mean change 0.0; ANCOVA analysis intervention $v$. control $F_{1,8}=5.55, P=0.04$, Cohen's $f=0.28$

Intervention mean change 1.2; control mean change-0.4; ANCOVA analysis intervention $v$. control $P=0.10$; Cohen's $f=0.35$

Intervention mean change 1.7; control mean change -0.4; ANCOVA analysis intervention $v$. control $P=0.12$; Cohen's $f=0.67$

Intervention (baseline mean score $9.3 v$. week 12 mean score 13.2†); control (no detail); ANCOVA analysis intervention $v$. control $F_{1,13}=5.58$

Intervention (baseline mean score $7.2 v$. week 12 mean score 9.6†) control (no detail); ANCOVA analysis intervention $v$. control $F_{1,13}=2 \cdot 27$

Intervention $v$. control mean score at 12 weeks (46.8 v. 45.8) Intrusion errors intervention $v$. control at 12 weeks $(0.20 v .1 .27)$; $F_{1,23}=6.48$; Cohen's $f=0.51$

Intervention $v$. control mean score at 12 weeks $(9.4 v .8 .4)$ Intrusion errors intervention $v$. control at 12 weeks $(0.98 v$ v. 2.3), $F_{1,23}=3.35$, Cohen's $f=0.35$

Intervention $v$. Control mean score at 12 weeks $(9.3 v .9 .5)$ Intrusion errors intervention $v$. control at 12 weeks (0.98 v. 2.3), $F_{1,23}=3.35$, Cohen's $f=0.35$

Intervention $v$. control mean score at 12 weeks $(14.4 v .14 .2)$ Intrusion errors intervention $v$. control at 12 weeks $(0.88 v .2 \cdot 2)$ $F_{1,23}=2.94$, Cohen's $f=0.34$

$\mathrm{HF}$ (mean change 8.0 (SD 5.3) words per $\left.60 \mathrm{~s}^{\star * \star}\right)$; IF (mean change 5.1 (SD 3.1) words per $60 \mathrm{~s}^{\star \star \star}$ ), LF (mean change 1.2 (SD 2.7) words per $60 \mathrm{~s} \dagger$ )

Intervention mean difference 3.32 (sD 3.45); control mean difference 1.38 (SD 2.66)

Intervention: mean change 1.55 (SD 3.96); control mean change 1.98 (SD 3.13)

Intervention mean change at 24 months, 0.003 (SD 0.569); control mean change at 24 months -0.130 (SD 0.619 )

Intervention mean score 12.28 (SD 3.56); control mean score 10.82 (SD 2.62)

Intervention mean score 13.44 (SD 3.66); control mean score 10.25 (SD 3.42)

Intervention mean score (month 1, 19.42 (sD 3.49) - month 4, 17.46 (sD 4.52)); control mean score (month 1, 20.50 (sD 4.31) - month $4,19.38$ (SD 4.65)) 


\begin{tabular}{|c|c|c|c|c|c|}
\hline $\begin{array}{l}\text { NIA-AA cognitive } \\
\text { domain }\end{array}$ & Study & Intervention & Cognitive function measure used & Intervention group and control group results & $\begin{array}{l}\text { Between group } \\
\text { difference }\end{array}$ \\
\hline & & & Delayed verbal memory & $\begin{array}{l}\text { Intervention mean score (month } 1,4.85 \text { (SD 2.91) - month } 4,4.34 \\
\text { (SD 2.74)); control mean score (month 1,5.23 (SD 2.63) - month } 4 \text {, } \\
4.65 \text { (SD 2.79)) }\end{array}$ & $\ddagger$ \\
\hline & & & Recognition verbal memory & $\begin{array}{l}\text { Intervention mean score (month } 1,8.92 \text { (SD } 2.06) \text { - month } 4,8.38 \\
\text { (SD 2.30)); control mean score (month 1, } 9.00 \text { (SD 2.80) - month } 4 \text {, } \\
8.00(\text { (SD 2.55)) }\end{array}$ & - \\
\hline & & & Visual memory & $\begin{array}{l}\text { Intervention mean score (month } 1,11.58 \text { (sD 2.19) - month } 4,12.77 \\
\text { (sD 2.67); control mean score (month 1, } 11.50 \text { (sD 2.60) - month } 4 \text {, } \\
11.85 \text { (SD 1.95)) }\end{array}$ & $\ddagger$ \\
\hline \multirow[t]{14}{*}{$\begin{array}{l}\text { Executive } \\
\text { function }\end{array}$} & Lee et al. $(2013)^{(27)}$ & $\begin{array}{l}\text { Fish oil supplementation with } \\
\text { concentrated DHA +EPA } v \text {. } \\
\text { placebo }\end{array}$ & Digit symbol substitution & $\begin{array}{l}\text { Intervention (baseline mean score } 5.5,95 \% \mathrm{Cl} 3.723,7 \cdot 218 \text { - } \\
12 \text { months mean score } 5.5,95 \% \mathrm{Cl} 3 \cdot 723,7 \cdot 218 \text { ); control } \\
\text { (baseline mean score } 4.9,95 \% \mathrm{Cl} 3.254,6.634-12 \text { months } \\
\text { mean score } 4.9,95 \% \mathrm{Cl} 3.254,6.634 \text { ) }\end{array}$ & - \\
\hline & & & CDT & $\begin{array}{l}\text { Intervention (baseline mean score } 7 \cdot 3,95 \% \mathrm{Cl} 6.810,7.880 \text { - } \\
12 \text { months mean score } 7.8,95 \% \mathrm{Cl} 7.142,8.477 \text { ); control } \\
\text { (baseline mean score } 7.5,95 \% \mathrm{Cl} 6.935,7.969 \text { - } 12 \text { months } \\
\text { mean score } 7.8,95 \% \mathrm{Cl} 7.145,8.436 \text { ) }\end{array}$ & $\ddagger$ \\
\hline & & & $\begin{array}{l}\text { Executive function } Z \text { score } \\
\quad \text { (cumulative score of all tests used) }\end{array}$ & $\begin{array}{l}\text { Intervention (mean change } 0.52(\text { SD } 0.869) \dagger) \text {; control (mean change } \\
-0.238(0.683) \text { ) }\end{array}$ & $\ddagger$ \\
\hline & $\begin{array}{l}\text { Petersen et al. } \\
(2005)^{(31)}\end{array}$ & $\begin{array}{l}2000 \mathrm{IU} \text { vitamin } \mathrm{E}, 10 \mathrm{mg} \\
\text { donepezil or placebo }\end{array}$ & $\begin{array}{l}\text { Executive function } Z \text { score (digits } \\
\text { backwards test, symbol digit } \\
\text { modalities test and number - } \\
\text { cancellation test) }\end{array}$ & $\begin{array}{l}\text { Intervention ( } 6 \text { months } Z \text { score } 0.11 \text {, sD } 0.41 \S-36 \text { months } Z \text { score }- \\
0.19 \text {, sD } 0.48 \text { ); control ( } 6 \text { months } Z \text { score } 0.04 \text {, sD } 0.42- \\
36 \text { months } Z \text { score }-0.19 \text {, sD } 0.53 \text { ) }\end{array}$ & $\ddagger$ \\
\hline & $\begin{array}{l}\text { Horie et al. } \\
\qquad(2016)^{(37)}\end{array}$ & $\begin{array}{l}\text { Nutrition counselling and energy } \\
\text { restriction } v \text {. standard care }\end{array}$ & Trail making test, part B & $\begin{array}{l}\text { Intervention (mean change }-8 \cdot 6,95 \% \mathrm{Cl}-71 \cdot 6,54 \cdot 5) \text {; control } \\
\quad \text { (mean change } 5 \cdot 1,95 \% \mathrm{Cl}-58 \cdot 3,68 \cdot 6 \text { ) }\end{array}$ & $\ddagger$ \\
\hline & & & Phonemic fluency & $\begin{array}{l}\text { Intervention (mean change } 0 \cdot 1,95 \% \mathrm{Cl}-0.5,5 \cdot 1 \text { ); control } \\
\text { (mean change } 2 \cdot 0,95 \% \mathrm{Cl}-3 \cdot 1,7 \cdot 1 \text { ) }\end{array}$ & $\ddagger$ \\
\hline & & & Semantic fluency & $\begin{array}{l}\text { Intervention (mean change } 1.1,95 \% \mathrm{Cl}-1.4,3 \cdot 6 \text { ); control } \\
\text { (mean change } 1.9,95 \% \mathrm{Cl}-0.6,4 \cdot 4)\end{array}$ & $\ddagger$ \\
\hline & & & $\begin{array}{l}\text { Modified Wisconsin Card Sorting } \\
\text { Test }\end{array}$ & $\begin{array}{l}\text { Intervention (mean change } 0.4,95 \% \mathrm{Cl}-0.3,1.0 \text { ); control } \\
\quad \text { (mean change } 0.7,95 \% \mathrm{Cl}-0.1,1.4 \text { ) }\end{array}$ & $\ddagger$ \\
\hline & $\begin{array}{l}\text { Krikorian et al. } \\
\quad(2012)^{(12)}\end{array}$ & $\begin{array}{l}\text { High carbohydrate diet } v \text {. very low } \\
\text { carbohydrate }\end{array}$ & Trail making test, part $\mathrm{B}$ & $\begin{array}{l}\text { Intervention (pre-intervention mean score } 79.2 \mathrm{~s} v \text {. post intervention } \\
\text { mean score } 82.9 \mathrm{~s}, F_{1,20}=0.46 \text { ); control (no detail) }\end{array}$ & $\ddagger$ \\
\hline & $\begin{array}{l}\text { Bayer-Carter et al. } \\
\qquad(2011)^{(38)}\end{array}$ & $\begin{array}{l}\text { High fat/high Gl diet } v \text {. low fat/low } \\
\text { GI diet }\end{array}$ & $\begin{array}{l}\text { Trail making test, part B } \\
\text { Stroop colour word } \\
\text { test } \\
\text { Verbal fluency }\end{array}$ & $\begin{array}{l}\text { The authors did not include these data in their published paper, } \\
\text { merely stating no diet related changes in the text }\end{array}$ & $\ddagger$ \\
\hline & $\begin{array}{l}\text { De Jager et al. } \\
\qquad(2012)^{(25)}\end{array}$ & $\begin{array}{l}0.8 \mathrm{mg} \text { folic acid, } 0.5 \mathrm{mg} \text { vitamin } \\
\mathrm{B}_{12} \text { and } 20 \mathrm{mg} \text { vitamin } \mathrm{B}_{6} \mathrm{~V} \\
\text { placebo }\end{array}$ & $\begin{array}{l}\text { CLOX (subgroup analyses, with } \\
\text { baseline tHcy levels) }\end{array}$ & $\begin{array}{l}\text { The odds of a correctly drawn item from CLOX1, after controlling for } \\
\text { confounders (CLOX2 at follow-up, CLOX1 at baseline, age, } \\
\text { education, ApoE } \varepsilon 4 \text { status and sex), was } 30 \% \text { greater in those } \\
\text { receiving B-vitamins in comparison to placebo (OR =0.26) }\end{array}$ & * \\
\hline & $\begin{array}{l}\text { Desideri et al. } \\
\quad(2012)^{(34)}\end{array}$ & $\begin{array}{l}990 \mathrm{mg} \text { HF } v \text {. IF } v \text {. LF cocoa } \\
\text { flavanols per day }\end{array}$ & Trail making test, part B & $\begin{array}{l}\mathrm{HF}\left(\text { mean change }-29.2(\mathrm{sD} 8.0) \mathrm{s}^{\star \star \star}\right) \text {, IF (mean change }-22.8 \\
\left.\left.(\mathrm{SD} 5 \cdot 1) \mathrm{s}^{\star \star \star}\right) \text { LF (mean change } 3.8(\mathrm{sD} 16.3) \mathrm{s}\right)\end{array}$ & * \\
\hline & $\begin{array}{l}\text { Soininen et al. } \\
(2017)^{(33)}\end{array}$ & $\begin{array}{l}\text { Souvenaid, a } 125 \mathrm{ml} \text { once-a-day } \\
\text { drink } v \text {. control }\end{array}$ & NTB executive function $Z$ score & $\begin{array}{l}\text { Intervention mean change at } 24 \text { months }-0.145 \text { (SD } 0.445 \text { ); control } \\
\text { mean change at } 24 \text { months }-0.039 \text { (SD } 0.506 \text { ) }\end{array}$ & $\ddagger$ \\
\hline & $\begin{array}{l}\text { Phillips et al. } \\
\qquad(2015)^{(29)}\end{array}$ & $\begin{array}{l}625 \mathrm{mg} \text { EPA }+600 \mathrm{mg} \text { DHA } v \text {. } \\
\text { placebo }\end{array}$ & CLOX2 & $\begin{array}{l}\text { Intervention mean score (month 1, } 14.08 \text { (SD 0.89) - month 4, } 14.08 \\
\quad(\text { SD 14.08)); control mean score (month } 1,14.38 \text { (SD 0.75) - month } \\
\quad 4,14.27 \text { (SD 0.67)) }\end{array}$ & $\ddagger$ \\
\hline \multirow[t]{2}{*}{ Attention } & $\begin{array}{l}\text { Horie et al. } \\
\qquad(2016)^{(37)}\end{array}$ & $\begin{array}{l}\text { Nutrition counselling and energy } \\
\text { restriction } v \text {. standard care }\end{array}$ & Digit span forward & $\begin{array}{l}\text { Intervention (mean change }-0.4,95 \% \mathrm{Cl}-1 \cdot 1,0.3 \text { ); control } \\
\quad \text { (mean change } 0.1,95 \% \mathrm{Cl}-0.6,0.9 \text { ) }\end{array}$ & $\ddagger$ \\
\hline & & & Digit span backward & $\begin{array}{l}\text { Intervention (mean change } 0 \cdot 2,95 \% \mathrm{Cl}-0 \cdot 8,1 \cdot 2) \text {; control } \\
\quad \text { (mean change } 0.1,95 \% \mathrm{Cl}-0.9,1 \cdot 1)\end{array}$ & $\ddagger$ \\
\hline
\end{tabular}




\section{Writish Journal of Nutrition}

Table 3. Continued

\begin{tabular}{|c|c|c|c|c|c|}
\hline $\begin{array}{l}\text { NIA-AA cognitive } \\
\text { domain }\end{array}$ & Study & Intervention & Cognitive function measure used & Intervention group and control group results & $\begin{array}{l}\text { Between group } \\
\text { difference }\end{array}$ \\
\hline & & & Trail making test, part $\mathrm{A}$ & $\begin{array}{l}\text { Intervention (mean change }-6 \cdot 195 \% \mathrm{Cl}-22 \cdot 6,10 \cdot 4) \text {; control } \\
\quad \text { (mean change }-0.7,95 \% \mathrm{Cl}-17 \cdot 3,15 \cdot 9)\end{array}$ & $\ddagger$ \\
\hline & Lee et al. $(2013)^{(27)}$ & $\begin{array}{l}\text { Fish oil supplementation with } \\
\text { concentrated DHA + EPA } v \text {. } \\
\text { placebo }\end{array}$ & CDT & 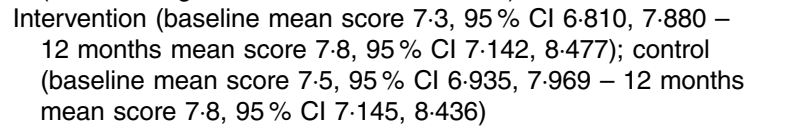 & $\ddagger$ \\
\hline & & & Digit span forward test & $\begin{array}{l}\text { Intervention (baseline mean score 8.0, } 95 \% \mathrm{Cl} 6.99,9.04- \\
12 \text { months mean score } 9.6,95 \% \mathrm{Cl} 8.437,10.749 \text { ); control } \\
\text { (baseline mean score } 8.5,95 \% \mathrm{Cl} 7.554,9.529-12 \text { months } \\
\text { mean score } 8.0 \text {, } \\
95 \% \mathrm{Cl} 6.877,9.113 \text { ) }\end{array}$ & * \\
\hline & & & Attention $Z$ score & $\begin{array}{l}\text { Intervention (mean change } 0.52(\text { sD } 0.869) \dagger) \text {; control (mean change } \\
-0.238(0.683))\end{array}$ & $\ddagger$ \\
\hline & $\begin{array}{l}\text { Desideri et al. } \\
{(2012)^{(34)}}^{(20)}\end{array}$ & $\begin{array}{l}990 \mathrm{mg} \mathrm{HF} v \text {. IF } v \text {. LF cocoa } \\
\text { flavanols per day }\end{array}$ & Trail making test, part $\mathrm{A}$ & $\begin{array}{l}\mathrm{HF}\left(\text { mean change }-14.3(\mathrm{sD} 4 \cdot 2) \mathrm{s}^{\star \star \star}\right) \text {, IF (mean change -8.8 (SD } \\
\left.\text { 3.4) } \mathrm{s}^{\star \star \star}\right) \text {, LF (mean change } 1.1 \text { (SD 13.0) s) }\end{array}$ & * \\
\hline & $\begin{array}{l}\text { Zhang et al. } \\
\qquad(2017)^{(30)}\end{array}$ & $2 \mathrm{~g} / \mathrm{d}$ DHA v. placebo & Digit span & $\begin{array}{l}\text { Intervention mean score } 13.44 \text { (SD 3.66); control mean score } 10.25 \\
\text { (SD 3.42) }\end{array}$ & * \\
\hline & $\begin{array}{l}\text { Philips et al. } \\
\qquad(2015)^{(29)}\end{array}$ & $\begin{array}{l}625 \mathrm{mg} E P A+600 \mathrm{mg} \text { DHA } v \text {. } \\
\text { placebo }\end{array}$ & Basic attention & $\begin{array}{l}\text { Intervention mean score (month } 1,6.38 \text { (SD 1.47) - month 4, } 6.54 \\
\text { (SD 1.33); control mean score (month 1, 6.65 (1.36) - month } 4 \text {, } \\
6.77 \text { (SD 1.31)) }\end{array}$ & $\ddagger$ \\
\hline \multirow[t]{3}{*}{ Language } & $\begin{array}{l}\text { Horie et al. } \\
\qquad(2016)^{(37)}\end{array}$ & $\begin{array}{l}\text { Nutrition counselling and energy } \\
\text { restriction } v \text {. standard care }\end{array}$ & Semantic fluency & $\begin{array}{l}\text { Intervention (mean change } 1 \cdot 1,95 \% \mathrm{Cl}-1 \cdot 4,3 \cdot 6 \text { ); control (mean } \\
\text { change } 1.9,95 \% \mathrm{Cl}-0 \cdot 6,4.4)\end{array}$ & $\ddagger$ \\
\hline & & & Phonemic fluency & $\begin{array}{l}\text { Intervention (mean change } 0 \cdot 1,95 \% \mathrm{Cl}-0.5,5 \cdot 1 \text { ); control (mean } \\
\text { change } 2 \cdot 0,95 \% \mathrm{Cl}-3 \cdot 1,7 \cdot 1 \text { ) }\end{array}$ & $\ddagger$ \\
\hline & $\begin{array}{l}\text { Petersen et al. } \\
\qquad(2005)^{(31)}\end{array}$ & $\begin{array}{l}2000 \mathrm{IU} \text { vitamin } \mathrm{E}, 10 \mathrm{mg} \\
\text { donepezil or placebo }\end{array}$ & $\begin{array}{l}\text { Language } Z \text { score (Boston naming } \\
\quad \text { test and category fluency test) }\end{array}$ & $\begin{array}{l}\text { Intervention ( } 6 \text { months } Z \text { score } 0.07 \text {, sD } 0.23 \S-36 \text { months } Z \text { score }- \\
0.10 \text {, sD } 0.35 \text { ); control }(6 \text { months } Z \text { score } 0.03 \text {, sD } 0.23 \text { - } \\
36 \text { months }-0.08 \text {, sD } 0.33 \text { ) }\end{array}$ & $\ddagger$ \\
\hline \multirow[t]{5}{*}{ VS } & Lee et al. $(2013)^{(27)}$ & $\begin{array}{l}\text { Fish oil supplementation with } \\
\text { concentrated DHA + EPA } v \text {. } \\
\text { placebo }\end{array}$ & Matrix reasoning block design test & $\begin{array}{l}\text { Intervention (baseline mean score } 7 \cdot 6,95 \% \mathrm{Cl} 6 \cdot 37,8 \cdot 75 \text { - } \\
12 \text { months mean score } 7 \cdot 1,95 \% \mathrm{Cl} 6 \cdot 27,7 \cdot 96) ; \text { control (baseline } \\
\text { mean score } 7 \cdot 3,95 \% \mathrm{Cl} 6 \cdot 16,8.45-12 \text { months mean score } 7.9 \text {, } \\
95 \% \mathrm{Cl} 7.07,8 \cdot 71 \text { ) }\end{array}$ & $\ddagger$ \\
\hline & & & VS $Z$ score & $\begin{array}{l}\text { Intervention (mean change } 0.17 \text { (sD } 0.84) \text { ); control (mean change } \\
0.04(\mathrm{sD} 0.60) \text { ) }\end{array}$ & $\ddagger$ \\
\hline & $\begin{array}{l}\text { Petersen et al. } \\
\qquad(2005)^{(31)}\end{array}$ & $\begin{array}{l}2000 \mathrm{IU} \text { vitamin } \mathrm{E}, 10 \mathrm{mg} \\
\text { donepezil or placebo }\end{array}$ & VS $Z$ score (CDT) & $\begin{array}{l}\text { Intervention ( } 6 \text { month } Z \text { score } 0.03 \text {, sD } 0.34-36 \text { months } Z \text { score }- \\
0.12 \text {, sD } 0.37 \text { ); control }(6 \text { month } Z \text { score }-0.01 \text {, sD } 0.34- \\
36 \text { months } Z \text { score }-0.11 \text {, sD } 0.39 \text { ) }\end{array}$ & $\ddagger$ \\
\hline & Ma et al. $(2016)^{(24)}$ & Folic acid $(400 \mu \mathrm{g} / \mathrm{d}) v$. control & Block design test & $\begin{array}{l}\text { Intervention (baseline mean score } 9.77 \text { (sD } 5.41 \text { ) }-6 \text { months mean } \\
\text { score } 13.28 \text { (sD } 4.21) \text { ); control (baseline mean score } 9.93 \text { (SD } \\
2.273)-6 \text { months mean score } 11.33 \text { (SD } 3.11) \text { ) }\end{array}$ & * \\
\hline & $\begin{array}{l}\text { Zhang et al. } \\
\qquad(2017)^{(30)}\end{array}$ & $2 \mathrm{mg}$ DHA v. placebo & Block design test & $\begin{array}{l}\text { Intervention (baseline mean score } 10.25 \text { (SD } 5.30-12 \text { months mean } \\
\text { score } 11.19 \text { (SD 4.07); control (baseline mean score } 9.63 \text { (SD 2.46) } \\
-12 \text { months mean score } 10.43 \text { (SD 3.51)) }\end{array}$ & $\ddagger$ \\
\hline
\end{tabular}

RAVLT, Rey Auditory Verbal Learning Test; HVLT-R, Hopkins Verbal Learning Test - Revised; tHcy, total homocysteine; Gl, glycaemic index; aMCl, amnesic mild cognitive impairment; V-PAL, Verbal Paired Associates Learning; CVLT, California Verbal Learning Test; CDT, clock drawing test; NTB, neuropsychological test battery; CLOX, executive clock drawing task.

* California Verbal Learning Test; CDT, clock drawing test; NTB, neuropsychological test battery; CLOX, exec

† Statistically significant difference $P \leq 0.05$ within group.

$\ddagger$ No statistically significant difference between intervention and control at study completion.

\& Statistically signilicanterenter

${ }_{* \star \star}$ Statistically significant difference $P \leq 0.001$ within group. 
treatment group and placebo ${ }^{(25)}$. In another B vitamin study, investigating folic acid alone ( $400 \mu \mathrm{g}$ daily for 6 months) $v$. conventional treatment ${ }^{(24)}$ results showed for short term memory that the intervention group had a significant increase in score from baseline to 6 months in comparison to the control $(P \leq 0 \cdot 001)$. Results also indicated that elevated homocysteine levels at baseline were associated with significantly poorer cognitive performance at intervention completion for the intervention group in comparison to the control ${ }^{(24)}$.

Vitamin E supplementation (2000 IU daily for 2 years) ${ }^{(31)}$, the medical food, Souvenaid containing the specific nutrition combination Fortasyn Connect $\left(125 \mathrm{ml}\right.$ daily) ${ }^{(33)}$ and chromium picolinate (CrPic) supplementation $(1000 \mu \mathrm{g}$ daily for 12 weeks) ${ }^{(32)}$ had no significant improvement in comparison to placebo for memory. Supplementation with CrPic showed significantly reduced intrusion errors, with the intervention group making significantly fewer errors on California Verbal Learning Test (CVLT) for learning $(P=0 \cdot 01)$ than the placebo group, however there was no significant reduction for recall and recognition memory ${ }^{(32)}$. In an investigation of the effects of a high carbohydrate diet ( $50 \%$ of total energy content) $v$. a very low carbohydrate $(5-10 \%$ of total energy content) diet in participants with $\mathrm{MCI}^{(12)}$, pre-intervention carbohydrate levels were recorded as $207 \mathrm{~g}$ for those in the 'high' carbohydrate group and $190 \mathrm{~g}$ in the 'low' carbohydrate group. Post-intervention carbohydrate levels measured $197 \mathrm{~g}$ for the 'high' carbohydrate group and $34 \mathrm{~g}$ for the 'low' carbohydrate group. These figures indicate that those in the 'low' group had a major dietary change whereas the 'high' group could be regarded as a control. Results showed no significant effect of the intervention for memory performance (brief visuospatial memory test, story recall and word list) between intervention and control groups ${ }^{(12)}$. Concord grape juice $^{(35)}$ (daily consumption between 6 and $9 \mathrm{ml} / \mathrm{kg}$ for 12 weeks) significantly improved verbal learning compared with the placebo $(P=0 \cdot 04)$. However, there were no significant differences between those consuming the grape juice and placebo for delayed verbal recall and spatial memory ${ }^{(35)}$. Furthermore, wild blueberry juice ${ }^{(36)}$ (daily consumption between 6 and $9 \mathrm{ml} / \mathrm{kg}$ for 12 weeks) had a significant improvement from baseline score to 12 weeks for verbal paired associates learning (V-PAL) cumulative learning $(P=0.009)$. In addition, mean scores for CVLT word list recall improved significantly within the intervention group from baseline to 12 weeks $(P=0 \cdot 04)$. There was a significant difference in V-PAL score between intervention and control groups $(P=0.03)$, however no significant difference was observed for CVLT performance between groups ${ }^{(36)}$.

Executive function. The domain of executive function was measured by twelve tests (Table 3). For this cognitive domain, measured within nine studies (56\%), two RCT showed a statistically significant improvement between groups at study completion $^{(25,34)}$. At 24 months follow-up, the odds of a correctly drawn item from CLOX1 (an executive clock drawing task), after controlling for confounders (CLOX2 at follow-up, CLOX1 at baseline, age, education, ApoE $\varepsilon 4$ status and sex), was $30 \%$ greater in those receiving B-vitamins $v$. placebo $(P=0 \cdot 02)^{(25)}$. For cocoa flavonol supplementation ${ }^{(34)}$, better scores for trail making test, part $\mathrm{B}(P \leq 0.05)$ were reported among participants who received $\mathrm{HF}$ and $\mathrm{IF}$ treatments $v$, the LF group. In addition, the time required to complete the trail making task, B significantly changed during the duration of the study $(P \leq 0 \cdot 0001)$. However, DHA +EPA supplementation $^{(27,29)}$, nutritional counselling with energy restriction $^{(37)}$, high fat/high GI $v$. low fat/low GI diet ${ }^{(38)}$, high carbohydrate $v$. low carbohydrate $\operatorname{diet}^{(12)}$, supplementation with Fortasyn Connect (Souvenaid) ${ }^{(33)}$ and vitamin $\mathrm{E}^{(31)}$ showed no significant difference in cognitive function tests between groups at study completion. There was a significant improvement in comparison with placebo at 6 months for those consuming vitamin E supplements $(P<0 \cdot 05)^{(31)}$. However, thereafter, this significant difference was not maintained beyond this time point.

Attention. As shown in Table 3, five of the sixteen (31\%) included studies measured the domain of attention. Nutritional counselling $v$. standard care showed no significant change in attention between groups after 12 months ${ }^{(37)}$. Whereas, cocoa flavonol supplementation ${ }^{(34)}$, significantly better scores for trail making test, part A $(P \leq 0.05)$ were reported among participants who received $\mathrm{HF}$ and IF treatments in comparison to the LF group. In addition, the time required to complete the trail making task, part A significantly changed during the duration of the study $(P \leq 0 \cdot 0001)^{(34)}$. DHA + EPA supplementation ${ }^{(27)}$ (one study) showed a significant improvement in digit span score from baseline to 12 months in the fish oil group $v$. placebo $(P \leq 0 \cdot 0001)^{(27)}$. However, there was no significant treatment effect reported between the fish oil and placebo groups for any of the other measures of attention ${ }^{(27)}$. Supplementation with DHA only ${ }^{(30)}$ showed significant improvements in digit span score in comparison to the placebo $(P \leq 0.0001)$. However, a third study with DHA+EPA supplementation ${ }^{(29)}$ found no significant differences between groups for attention.

Language. In all, two of the sixteen (13\%) studies measured the cognitive domain of language (Table 3 ). There were no significant differences between groups for nutritional counselling with energy restriction ${ }^{(37)}$. For vitamin E supplementation ${ }^{(31)}$, there was a significant difference in score from the baseline value between groups at 6 months $(P \leq 0.05), 12$ months $(P \leq 0.05)$ and 18 months $(P \leq 0.05)$, however, thereafter this significant difference was not maintained until intervention completion (36 months) $)^{(31)}$.

Visuospatial skills. In all, four studies (25\%) measured the cognitive domain of visuospatial skills (Table 3). Supplementation with folic acid was the only study to show a significant interaction effect between groups for visuospatial skills $(P=0.03)^{(24)}$. In addition, higher baseline homocysteine levels were associated with poorer cognitive performance on the block design test at the end of the intervention in comparison with the placebo (estimate value $=-0.079, P \leq 0 \cdot 001)^{(24)}$. Fish oil supplementation with concentrated $\mathrm{DHA}+\mathrm{EPA}^{(27)}, \mathrm{DHA}^{(30)}$ 
or vitamin E supplementation ${ }^{(31)}$ did not show any significant differences between groups.

Global cognitive function. For cocoa flavonol supplementation $^{(34)}$ (online Supplementary Table S2), there was no significant change in MMSE score between the HF, IF or LF treatment groups over the duration of the study $(P=0 \cdot 13)$. However, results also showed that the composite cognitive $Z$ score significantly changed during the study $(P \leq 0 \cdot 0001)$. The cognitive $Z$ score at the end of the study follow-up was significantly $(P \leq 0.05)$ better in the HF group in comparison to the LF group ${ }^{(34)}$. Vitamin B supplementation ${ }^{(25)}$ indicated no significant effect of treatment $(P=0.57)$ on global cognition as measured by MMSE. However, analysis did show that those who had high baseline concentrations of homocysteine and were treated with $\mathrm{B}$ vitamins, were 1.58 more likely to provide a correct answer on the MMSE test than the placebo group $(P<0 \cdot 001)$. However, there was no significant difference for those with low baseline homocysteine, between the $\mathrm{B}$ vitamin or placebo groups. Similarly, fish oil supplementation ${ }^{(27)}$ (one study) showed no statistically significant differences between groups for cognitive function as measured by the MMSE. Furthermore, vitamin E supplementation ${ }^{(31)}$ at 6 months intervention showed a significant difference in comparison with placebo for overall cognitive function calculated by a composite $Z$ score $(P \leq 0 \cdot 01)$. However, at 36 months this significant difference between groups was not maintained.

\section{Assessment of methodological quality and risk of bias}

The quality ${ }^{(22)}$ of the sixteen included studies varied, with eight studies achieving the maximum total score of $5^{(25-28,30,31,33,34)}$ (online Supplementary Table S3). Thus, it was deemed that these studies stated appropriate randomisation processes, were clearly indicated as double blinded and the authors accounted for any participant withdrawals during the study. In all, two studies ${ }^{(12,38)}$ scored one on the Jadad scale ${ }^{(22)}$ and stated that participants were randomised however did not specify the randomisation process, if double-blinding took place and if any participant withdrawals occurred. Low risk of bias scores ${ }^{(23)}$ were allocated for selection bias $\left(\begin{array}{ll}n & 9\end{array}\right)^{(24-28,30,31,33,34)}$, performance bias $\left(\begin{array}{ll}n & 7\end{array}\right)^{(25,26,28-30,33,34)}$, attrition $\left(\begin{array}{ll}n & 9\end{array}\right)^{(24,25,27-30,33,34,37)}$ and detection bias $(n 6)^{(24,26,30,33,34,37)}$ (online Supplementary Table S4). A high risk score was documented for detection bias ( $n$ $3)^{(12,38)}$ and performance bias $(n 2)^{(12)}$ as there were no details provided of any double blinding method used.

\section{Discussion}

The aim of the present systematic review was to examine the effect of diet, either alone or in combination with lifestyle and/ or cognitive strategies, on cognitive health outcomes in patients with MCI. Together with the limited number of RCT conducted and the heterogeneity of the studies in this review, a narrative synthesis of the findings was implemented. Studies varied greatly in terms of the nature of dietary intervention and cognitive outcome measures used. Furthermore, there were no studies that measured the effectiveness of lifestyle and/or cognitive strategies in combination with their dietary intervention. Overall, it was evident that the findings were inconsistent across the studies and do not provide clear evidence to support the effect of any specific diet or dietary component on cognition in MCI patients.

Diet has been suggested to have a significant association with cognitive decline and progression to dementia, particularly showing a protective role against the harmful effects of neuroinflammation and oxidative stress ${ }^{(40)}$. Although the pathways related to their role are complex and variable throughout the literature $^{(14-16,41)}$ it is thought that antioxidants in foods such as fruit and vegetables help to reduce oxidative stress levels in the brain and $n-3$ PUFA in foods such as oily fish, are additionally linked to reduced inflammation ${ }^{(8)}$. There are plausible suggestions to support these mechanisms by the results of this review. There were some improvements in cognitive function, particularly in the domain of memory, reported for polyphenol compounds (e.g. cocoa flavonols ${ }^{(34)}$ ), fish oil supplementation with concentrated $\mathrm{DHA}+\mathrm{EPA}^{(27,28)}$ or DHA alone ${ }^{(30)}$ and beverages which are high in these bioactive, antioxidant properties e.g. concord grape juice ${ }^{(35)}$ and wild blueberry juice $^{(36)}$. However, some of these studies either had small, potentially underpowered sample sizes, used a limited number of cognitive tests to measure outcomes or had shorter intervention durations therefore these results should be interpreted with caution.

\section{Nutrient and food supplementation}

As mentioned, antioxidant compounds such as vitamins A, C and $\mathrm{E}$ have a role in regulation of oxidative stress, a pathway linked with neurodegeneration and cognitive decline ${ }^{(42)}$. However in this review, diet supplementation with vitamin $\mathrm{E}^{(31)}$ had no significant effect on progression from MCI to dementia and/or $\mathrm{AD}$ or on cognitive function at intervention completion. Furthermore, meta-analyses have reported no significant effect of vitamin $\mathrm{E}$ on cognitive function outcomes $^{(43,44)}$. The particular form of vitamin $\mathrm{E}$ used could have an influence on the impact of this nutritional component on cognitive decline, with research suggesting total tocopherol plasma concentrations rather than single tocopherols may be more valuable at predicting cognitive impairment, particularly $\mathrm{AD}^{(45)}$. Furthermore, as we consume foods in complex patterns, resulting in ingestion of combinations of various forms of vitamin E, it may be more beneficial to focus research efforts away from single forms and follow a more holistic investigation $^{(15)}$. In this review, supplementation with cocoa flavonols ${ }^{(34)}$ showed better cognitive performances for those who received higher flavonols concentrations compared with lower concentrations. There are suggestions in the literature that flavonoids may exert their neuroprotective properties in a similar mechanism to antioxidants in the body ${ }^{(46)}$. However, further indications suggest that flavonoids may have a more prominent role in the regulation of neuronal signalling pathways $^{(47)}$ or neuro-inflammation ${ }^{(48)}$. It is clear that further research is required to fully explore the mechanism of action of 
flavonoid compounds and investigate the potential role they may have in protecting against cognitive decline ${ }^{(49)}$.

Low folate and B vitamin status is linked to cognitive dysfunction during the ageing process and better cognitive performances have been associated with higher intakes of $\mathrm{B}$ vitamins $^{(50-52)}$. Furthermore, increased levels of homocysteine have been linked to poorer cognition, particularly in memory and attention ${ }^{(53-55)}$. This may be explained by the role that $\mathrm{B}$ vitamins have in one-carbon metabolic pathways in the body, acting as co-factors for the remethylation of homocysteine to methionine, producing the methyl-donor, $S$ adenosylmethionine. This methyl donor has a specific role in the methylation of phospholipids and neurotransmitters in the brain, thus indicating how a depletion in B vitamins status may influence cognitive function and ultimately, cognitive impairment ${ }^{(56,57)}$. In this review, supplementation with a $\mathrm{B}$ vitamin $_{\text {combination }}^{(25)}$ or with folic acid alone ${ }^{(24)}$ had significant effects on executive function ${ }^{(25)}$ and furthermore, when baseline homocysteine levels were elevated, there were significant improvements in global cognition ${ }^{(25)}$, memory ${ }^{(24,25)}$ and visuospatial skills ${ }^{(24)}$. In support, not only have improvements been observed in performance based cognitive tests, $\mathrm{B}$ vitamin supplementation (folic acid, vitamin $\mathrm{B}_{6}$ and $\mathrm{B}_{12}$ combination) have resulted in reduced rates of brain atrophy in $\mathrm{MCI}^{(58,59)}$; a process which could result in progression to AD if allowed to advance. However, findings are mixed with metaanalyses of clinical trial data reporting no significant effect of $B$ vitamins on cognitive function ${ }^{(43,60)}$. Therefore, further trial research is warranted to confirm the role of $\mathrm{B}$ vitamins in reducing cognitive decline.

PUFA have been associated with promoting cognitive function, primarily as a result of their anti-inflammatory properties $^{(61)}$. Furthermore, $n-3$ fatty acids, particularly DHA, are a key component of neuronal membranes in the brain, influencing neurogenesis and neuronal function ${ }^{(41,62)}$. In this review, supplementation with $\mathrm{DHA}+\mathrm{EPA}^{(27,28)}$ reported significant improvements in the domain of memory, with DHA supplementation alone ${ }^{(30)}$ showing an additional improvement in attention, albeit by a single cognitive test. In contrast, evidence from meta-analyses have reported no significant effect of $n-3$ fatty acids on cognitive outcomes ${ }^{(43,62)}$. Furthermore, it has been suggested that fatty acid supplementation in individuals who are homozygous carriers of the ApoE $\varepsilon 4$ allele, a risk factor for cognitive decline, could be resistant from the potential protective effects of fatty acids on cognitive health ${ }^{(63)}$. Thus, this is an important covariate to consider when designing trials to test effectiveness of fatty acid supplementation. However, some observational evidence does exist to support the role of $n-3$ fatty acids in promoting cognition with a study that followed non-demented participants for 4 years, finding higher plasma EPA concentrations to be associated with a lower incidence of dementia ${ }^{(64)}$. In addition, an intervention study with older adults with subjective memory impairment investigated fatty acid supplementation $(\mathrm{EPA}+\mathrm{DHA}) \quad v$. maize oil placebo ${ }^{(65)}$. Results showed significantly improved cortical blood oxygen level-dependent activity during a working memory task in the fish oil group compared with placebo. In this review, one study investigating
$\mathrm{DHA}+\mathrm{EPA}$ supplementation ${ }^{(29)}$ found no effect on cognitive function in comparison to control. A plausible explanation for this finding could be that the placebo used this study was olive oil, a component of the Mediterranean diet associated with improved cognitive function owing to its anti-inflammatory properties $^{(66)}$. Therefore, further investigation of the role of fatty acids and cognitive decline is justified through well-designed, robust studies.

\section{Whole-foods/dietary patterns}

Only three of the sixteen studies included in this review ${ }^{(12,37,38)}$, focused their diet intervention on 'whole-foods/dietary patterns' rather than single-nutrient supplements or single food products. In everyday situations, individuals consume holistic dietary patterns which involve complex interactions between nutrients ${ }^{(67)}$. It therefore could be suggested that the more representative intervention design to measure the effects of diet on cognition could be that which involved a dietary pattern rather than focused on a single nutrient. In this review, however, these studies were heterogeneous in terms of the dietary intervention and reported mixed findings. Research evidence suggests that ketogenic diets ${ }^{(68)}$ and energy restriction ${ }^{(69)}$ may have a promising, yet under-investigated, role in $\mathrm{AD}$ prevention, suggesting links to brain glucose metabolism ${ }^{(68)}$, reduction in oxidative stress $^{(69)}$ and anti-inflammatory mechanisms ${ }^{(69)}$. There is also emerging evidence from observational studies to suggest a protective role for healthy dietary patterns such as the Mediterranean diet on MRI measured brain structures ${ }^{(70-72)}$ and therefore further investigation of such dietary patterns is necessary, with the inclusion of more rigorous assessment measures, to help to provide insight into potential mechanisms of how diet can impact brain health.

\section{Use of biomarkers and cognitive markers}

CSF biomarkers may be a valuable asset in detecting pathological changes in neurological diseases, owing to the processes of extracellular amyloid- $\beta$ deposition and accumulation of hyperphosphorylated tau proteins ${ }^{(73)}$. One study ${ }^{(38)}$ in this review included biomarker analysis in addition to cognitive test measures. Increased concentrations of CSF A $\beta 42$ were observed in those with a MCI consuming a low diet (low saturated fat/low GI) in comparison to healthy controls who observed a decrease in CSF A $\beta 42$ levels (online Supplementary material). Thus, CSF biomarkers in this study changed in response to diet in aMCI patients in the absence of any discernible changes in cognitive function test scores, albeit in very small sample. These differences could provide insights into the mechanisms of action of $\beta$-amyloid in the body in cognitive impairment. In particular, biomarker analysis may be more sensitive to dietary changes and could be an important consideration for future dietary intervention studies as the use of biomarkers could be a more rigorous approach to assess cognitive performance in this patient group ${ }^{(74)}$. Furthermore, it has been suggested that the use of brain imaging as a cognitive marker such as MRI scanning is a more robust measure of cognition in comparison to questionnaire based tests ${ }^{(50,75)}$. In all, three studies in this 
review reported on cognitive marker information, including $\operatorname{MRI}^{(30,33)}$ and functional MRI imaging ${ }^{(32)}$, as an additional outcome measure for cognitive function, depicting some significant interaction effects for the intervention group that were not entirely reflected by cognitive function tests (online Supplementary material). Brain imaging techniques have been used in nutrition and cognition research, with investigations into B vitamins utilising MRI scanning to detect changes in brain atrophy in $\mathrm{MCI}^{(58,59)}$, functional MRI scanning to explore fish oil supplementation in older adults with subjective memory impairment ${ }^{(65)}$ as well as investigations of $\beta$-amyloid load using positron emission tomography (PET) and neuronal activity via PET imaging with 2-[ ${ }^{18}$ F fluoro-2-deoxy-Dglucose $^{(76)}$. Therefore, the use of these higher quality methods could be implemented in future dietary intervention trials to comprehensively measure the potential effects of diet on cognition and explore mechanisms.

The mixed evidence found on the effect of diet on cognition among MCI participants may be explained by the heterogeneity of studies included, owing to variation in cognitive outcome measures used, differences in the diet intervention type (supplements $v$. single food products $v$. dietary patterns), variations in sample size and duration of intervention. Furthermore, the small number of dietary intervention studies conducted among this patient group make it difficult to provide conclusive evidence to support the effect of diet on cognitive outcomes. Of the sixteen included studies, those with $\mathrm{B}$ vitamin and/or folic acid supplementation $^{(24,25)}$, DHA/EPA supplementation ${ }^{(27,28,30)}$ or cocoa flavonol rich drinks ${ }^{(34)}$ appeared to have the most consistent effects on cognitive outcomes. However, it is difficult to confirm that these dietary interventions are the most effective in terms of promoting cognitive function due to the low number of studies testing the same intervention. Nonetheless, the outcomes of the systematic review highlight the need for well-designed, robust RCT, with pretested and informed methodological characteristics to further explore the role of diet in cognitive decline.

\section{Limitations}

During the literature search for this review, a broad search strategy was used to ensure the search covered all related aspects to the reviews aims and objectives. However, search limitations were set to only include studies in English language and the grey literature was not included for this review, therefore this could have resulted in language and publication bias. As RCT were the study design of choice for inclusion, this may have caused selection bias. However, as RCT are considered the best design for assessing the effect of a dietary intervention with their ability to identify causality ${ }^{(77)}$, this therefore provides justification for the decision. Pilot studies were not included in this review, as these studies are likely to have an underpowered sample size. The number of studies included in this review were small, however, as there are few RCT completed in this area, this supports the need for further intervention studies to increase the evidence-base. Due to the heterogeneity of the included studies, the data were not meta-analysed. Instead, a rigorous narrative review was implemented. Study characteristics, such as short study durations, may have not provided sufficient time to view a change in cognitive outcomes. It has been suggested that long term, RCT are the best approach in the design of a nutritional intervention to measure cognitive performance, with estimations that the most effect preventative trials require up to 3-5 years duration and follow-up ${ }^{(78)}$. Furthermore, ensuring a sufficient sample size though determination by a power calculation will provide a more stringent approach to the research design. Therefore, it is important when designing intervention studies that duration and sample size are pre-tested, though a feasibility study or by comparison to similar studies in the field.

In all, eight of the sixteen studies in this review achieved the maximum quality score as assessed by the Jadad scale ${ }^{(22)}$. Those studies who received the lowest scores failed to provide details on the randomisation and blinding processes which took place in the study. It is important to note however, as both studies involved a dietary pattern intervention rather than a supplement/placebo, it is impractical to ensure participants and researchers are blinded to the intervention group. Therefore, the decision that these studies are of 'low quality' is difficult to confirm. Furthermore, for risk of bias, a number of studies were allocated uncertain risk for selection, performance, attrition and detection bias due to inadequate information on randomisation, double blinding and/or withdrawals. Finally, a challenge within this review was the heterogeneity of cognitive outcome measures used to determine cognitive change. Some studies grouped results by domain, while others by the single cognitive tests used. This made it difficult when presenting the results of this review, as some study results did not exactly fit within the cognitive domains, as these were not specified in the original paper. In line with the NIA-AA criteria for the diagnosis of $\mathrm{MCI}^{(3)}$, which state that for a diagnosis of MCI individuals must have deterioration in one or more cognitive domains, it would be beneficial for analysis purposes if future intervention studies could assess cognition based on these domains to allow better comparison of results. However, in saying that, even the tests used to measure cognition within domains vary greatly and there is a lack of standardisation. It is evident therefore, that there is a demand to determine a specialised cognitive test battery that can be used to measure change in cognition, particularly within an MCI population. Furthermore, change in cognition requires time, more rigorous examinations and evaluation by clinical specialist ${ }^{(79)}$. These are all important considerations for future intervention trials going forward.

\section{Conclusion}

To date there is insufficient RCT evidence on the effect of whole diets or specific dietary components on cognitive outcomes in MCI patients. Existing studies are heterogeneous in terms of the dietary intervention, duration, sample size and cognitive outcome measures assessed, with the most consistent results for cognitive function shown by B vitamins, folic acid, DHA and/or EPA and cocoa flavonol supplementation. Further exploration of the potential beneficial effect of diet on cognitive outcomes in MCI is merited. 


\section{Acknowledgements}

This research received no specific grant from any funding agency or from commercial or not-for-profit sectors.

The authors' responsibilities were as follows: A. M. M. had the primary responsibility of writing the manuscript, A. M. M. conducted the literature search; A. M. M. and C. T. M. conducted and reviewed the data extraction; J. V. W., B. M., C. T. M. and M. C. M. reviewed and approved the manuscript. All authors were involved in editing and revisions of the article.

The authors declare no conflicts of interest arising from the conclusions of this work.

\section{Supplementary material}

For supplementary material/s referred to in this article, please visit https://doi.org/10.1017/S0007114518002945

\section{References}

1. Peracino A \& Pecorelli S (2016) The epidemiology of cognitive impairment in the aging population: implications for hearing loss. Audiol Neurotol 21, 3-9.

2. Deary IJ, Corley J, Gow AJ, et al. (2009) Age-associated cognitive decline. Br Med Bull 92, 135-152.

3. Albert MS, DeKosky ST, Dickson D, et al. (2011) The diagnosis of mild cognitive impairment due to Alzheimer's disease: recommendations from the National Institute on Aging-Alzheimer's Association workgroups on diagnostic guidelines for Alzheimer's disease. Alzheimers Dement 7, 270-279.

4. Roberts R \& Knopman DS (2013) Classification and epidemiology of MCI. Clin Geriatr Med 29, 753-772.

5. Tschanz JT, Welsh-Bohmer KA, Lyketsos CG, et al. (2006) Conversion to dementia from mild cognitive disorder: the Cache County Study. Neurology 67, 229-234.

6. Martin M, Clare L, Altgassen AM, et al. (2011) Cognition-based interventions for healthy older people and people with mild cognitive impairment. The Cochrane Database of Systematic Reviews 2011, issue 1 CD006220.

7. Cherbuin N, Anstey KJ \& Baune BT (2017) Oxidative stress, inflammation and mild cognitive impairment. Eur Psychiatry 41, S742.

8. Alles B, Samieri C, Feart C, et al. (2012) Dietary patterns: a novel approach to examine the link between nutrition and cognitive function in older individuals. Nutr Res Rev 25, 207-222.

9. Dauncey MJ (2009) New insights into nutrition and cognitive neuroscience. Proc Nutr Soc 68, 408-415.

10. Gomez-Pinilla F \& Tyagi E (2013) Diet and cognition: interplay between cell metabolism and neuronal plasticity. Curr Opin Clin Nutr Metab Care 16, 726-733.

11. Smith PJ, Blumenthal JA, Babyak MA, et al. (2010) Effects of the dietary approaches to stop hypertension diet, exercise, and caloric restriction on neurocognition in overweight adults with high blood pressure. Hypertension 55, 1331-1338.

12. Krikorian R, Shidler MD, Dangelo K, et al. (2012) Dietary ketosis enhances memory in mild cognitive impairment. Neurobiol Aging 33, 425.e19-425.e27.

13. Valls-Pedret C, Sala-Vila A, Serra-Mir M, et al. (2015) Mediterranean diet and age-related cognitive decline: a randomized clinical trial. JAMA 175, 1094-1103.

14. Frisardi V, Panza F, Seripa D, et al. (2010) Nutraceutical properties of Mediterranean diet and cognitive decline: possible underlying mechanisms. J Alzheimer's Dis 22, 715-740.
15. Caracciolo B, Xu W, Collins S, et al. (2014) Cognitive decline, dietary factors and gut-brain interactions. Mech Ageing Dev 136-137, 59-69.

16. Knight A, Bryan J \& Murphy K (2016) Is the Mediterranean diet a feasible approach to preserving cognitive function and reducing risk of dementia for older adults in Western countries? New insights and future directions. Ageing Res Rev 25, 85-101.

17. Milte CM \& McNaughton SA (2016) Dietary patterns and successful ageing: a systematic review. Eur J Nutr 55, 423-450.

18. Nagamatsu LS, Chan A, Davis JC, et al. (2013) Physical activity improves verbal and spatial memory in older adults with probable mild cognitive impairment: a 6-month randomized controlled trial. J Aging Res, 861-893.

19. Lautenschlager NT, Cox KL, Flicker L, et al. (2008) Effect of physical activity on cognitive function in older adults at risk for Alzheimer disease: a randomized trial. JAMA 300, 1027-1037.

20. Li H, Li J, Li N, et al. (2011) Cognitive intervention for persons with mild cognitive impairment: a meta-analysis. Ageing Res Rev 10, 285-296.

21. Centre for Reviews and Dissemination (CRD) (2009) Systematic Reviews: CRD's Guidance for Undertaking Reviews in Healthcare. York: University of York.

22. Jadad AR, Moore RA, Carroll D, et al. (1996) Assessing the quality of reports of randomized clinical trials: is blinding necessary? Contemp Clin Trials 17, 1-12.

23. Higgins JPT \& Green S (2011) Cochrane Handbook for Systematic Reviews of Interventions version 5.1.0 [updated March 2011]. The Cochrane collaboration. www.cochrane-handbook.org (accessed November 2016).

24. Ma F, Wu T, Zhao J, et al. (2016) Effects of 6-month folic acid supplementation on cognitive function and blood biomarkers in mild cognitive impairment: a randomized controlled trial in China. J Gerontol A Biol Sci Med Sci 1, 1376-1383.

25. de Jager CA, Oulhaj A, Jacoby R, et al. (2012) Cognitive and clinical outcomes of homocysteine-lowering B-vitamin treatment in mild cognitive impairment: a randomized controlled trial. Int. J. Geriatr. Psychiatry 27, 592-600.

26. DeKosky ST, Williamson JD, Fitzpatrick AL, et al. (2008) Ginkgo biloba for prevention of dementia: a randomized controlled trial. JAMA 300, 2253-2262.

27. Lee LK, Shahar S, Chin AV, et al. (2013) Docosahexaenoic acid-concentrated fish oil supplementation in subjects with mild cognitive impairment (MCI): a 12-month randomised, double-blind, placebo-controlled trial. Psychopharmacology 225, 605-612.

28. Bo Y, Zhang X, Wang Y, et al. (2017) The $n$-3 polyunsaturated fatty acids supplementation improved the cognitive function in Chinese elderly with mild cognitive impairment: a double blind randomised controlled trial. Nutrients 9, 1-11.

29. Phillips MA, Childs CE, Calder PC, et al. (2015) No effect of omega-3 fatty acid supplementation on cognition and mood in individuals with cognitive impairment and probable Alzheimer's disease: a randomised controlled trial. Int J Mol Sci 16, 24600-24613.

30. Zhang YP, Miao R, LI Q, et al. (2017) Effects of DHA supplementation on hippocampal volume and cognitive function in older adults with mild cognitive impairment: a 12 month randomised, double blind, placebo controlled trial. $J$ Alzheimers Dis 55, 497-507.

31. Petersen RC, Thomas RG, Grundman M, et al. (2005) Vitamin $\mathrm{E}$ and donepezil for the treatment of mild cognitive impairment. N Eng J Med 352, 2379-2388.

32. Krikorian R, Eliassen JC, Boespflug EL, et al. (2010) Improved cognitive-cerebral function in older adults with chromium supplementation. Nutr Neurosci 13, 116-122. 
33. Soininen H, Solomon A, Visser PJ, et al. (2017) 24-month intervention with a specific multinutrient in people with prodromal Alzheimer's disease (LipiDiDiet): a randomised, double-blind, controlled trial. Lancet Neurol 16, 965-975.

34. Desideri G, Kwik-Uribe C, Grassi D, et al. (2012) Benefits in cognitive function, blood pressure, and insulin resistance through cocoa flavanol consumption in elderly subjects with mild cognitive impairment novelty. Hypertension $\mathbf{6 0}$, 794-801.

35. Krikorian R, Nash TA, Shidler MD, et al. (2010) Concord grape juice supplementation improves memory function in older adults with mild cognitive impairment. Br J Nutr 103, 730-734.

36. Krikorian R, Shidler MD, Nash TA, et al. (2010) Blueberry supplementation improves memory in older adults. J Agric Food Chem 58, 3996-4000.

37. Horie NC, Serrao VT, Simon SS, et al. (2016) Cognitive effects of intentional weight loss in elderly obese individuals with mild cognitive impairment. J Clin Endocrinol Metab 101, 1104-1112.

38. Bayer-Carter JL, Green PS, Montine TJ, et al. (2011) Diet intervention and cerebrospinal fluid biomarkers in amnestic mild cognitive impairment. Arch Neurol 68, 743-752.

39. World Health Organization (2010) Global recommendations for physical activity on health. http://apps.who.int/iris/bitstream/10665/44399/1/9789241599979_eng.pdf （accessed June 2017).

40. Tang EYH, Harrison SL, Albanese E, et al. (2015) Dietary interventions for prevention of dementia in people with mild cognitive impairment. Cochrane Database Syst Rev, issue 10, CD011910.

41. Smith PJ \& Blumenthal JA (2016) Dietary factors and cognitive decline. J Prev Alzheimer's Dis 3, 53-64.

42. Morris MC (2012) Nutritional determinants of cognitive aging and dementia. Proc Nutr Soc 71, 1-13.

43. Forbes SC, Holroyd-Leduc JM, Poulin MJ, et al. (2015) Effect of nutrients, dietary supplements and vitamins on cognition: a systematic review and meta-analysis of randomized controlled trials. Can Geriatr J 18, 231-245.

44. Farina N, Llewellyn D, Isaac MGEKN, et al. (2017) Vitamin E for Alzheimer's dementia and mild cognitive impairment. Cochrane Database Syst Rev, issue 4, CD0028.

45. Mangialasche F, Kivipelto M, Mecocci P, et al. (2010) High plasma levels of vitamin $\mathrm{E}$ forms and reduced Alzheimer's disease risk in advanced age. J Alzheimer's Dis 20, 1029-1037.

46. Best T \& Dye L (2015) Nutrition for Brain Health and Cognitive Performance, pp. 251-261 [T Best and L Dye, editors]. London: CRC Press.

47. Vazour D, Vafeiadou K, Rodriguez-Mateos A, et al. (2008) The neuroprotective potential of flavonoids: a multiplicity of effects. Genes Nutr 3, 115-126.

48. Spencer JP (2009) Flavonoids and brain health: multiple effects underpinned by common mechanisms. Genes Nutr $\mathbf{4}$, 243-250.

49. Bell L, Lamport DJ, Butler LT, et al. (2015) A review of the cognitive effects observed in humans following acute supplementation with flavonoids, and their associated mechanisms of action. Nutrients 7, 10290-10306.

50. Porter K, Hoey L, Hughes C, et al. (2016) Causes, consequences and public health implications of low B-vitamin status in ageing. Nutrients $\mathbf{8}, 725$.

51. Morris MS, Selhub J \& Jacques PF (2012) Vitamin B-12 and folate status in relation to decline in scores on the Mini-Mental State Examination in the Framingham Heart Study. $J$ Am Geriatr Soc 60, 1457-1464.
52. Tangney CC, Tang Y, Evans DA, et al. (2009) Biochemical indicators of vitamin $\mathrm{B}_{12}$ and folate insufficiency and cognitive decline. Neurology 72, 361-367.

53. Faux NG, Ellis KA, Porter L, et al. (2011) Homocysteine, vitamin $B_{12}$, and folic acid levels in Alzheimer's disease, mild cognitive impairment, and healthy elderly: baseline characteristics in subjects of the Australian Imaging Biomarker Lifestyle study. J Alzheimer's Dis 27, 909-922.

54. Kado DM, Karlamangla AS, Huang MH, et al. (2005) Homocysteine versus the vitamins folate, $\mathrm{B}_{6}$, and $\mathrm{B}_{12}$ as predictors of cognitive function and decline in older high-functioning adults: MacArthur Studies of Successful Aging. Am J Med 118, 161-167.

55. Duthie SJ, Whalley LJ, Collins AR, et al. (2002) Homocysteine, $\mathrm{B}$ vitamin status, and cognitive function in the elderly. Am J Clin Nutr 75, 908-913.

56. Moore K, O'Shea M, Hughes CF, et al. (2017) Current evidence linking nutrition with brain health in ageing. Nutr Bull $\mathbf{4 2}$, 61-68.

57. Smith AD \& Refsum H (2016) Homocysteine, B vitamins, and cognitive impairment. Annu Rev Nutr 36, 211-239.

58. Smith AD, Smith SM, de Jager CA, et al. (2010) Homocysteinelowering by $\mathrm{B}$ vitamins slows the rate of accelerated brain atrophy in mild cognitive impairment: a randomized controlled trial. PLoS One 5, e12244.

59. Douaud G, Refsum H, de Jager CA, et al. (2013) Preventing Alzheimer's disease-related gray matter atrophy by B-vitamin treatment. Proc Natl Acad Sci U S A 110, 9523-9528.

60. Clarke R, Bennett D, Parish S, et al. (2014) Effects of homocysteine lowering with $\mathrm{B}$ vitamins on cognitive aging: metaanalysis of 11 trials with cognitive data on 22,000 individuals. Am J Clin Nutr 100, 657-666.

61. Gillette-Guyonnet S, Secher M \& Vellas B (2013) Nutrition and neurodegeneration: epidemiological evidence and challenges for future research. Br J Clin Pharmacol 75, 738-755.

62. Sydenham E, Dangour AD \& Lim WS (2012) Omega 3 fatty acid for the prevention of cognitive decline and dementia. Cochrane Database Syst Rev, issue 6, CD00537.

63. Chouinard-Watkins R \& Plourde M (2014) Fatty acid metabolism in carriers of apolipoprotein E epsilon 4 allele: is it contributing to higher risk of cognitive decline and coronary heart disease? Nutrients 6, 4452-4471.

64. Samieri C, Feart C, Letenneur L, et al. (2008) Low plasma eicosapentaenoic acid and depressive symptomatology are independent predictors of dementia risk. Am J Clin Nutr $\mathbf{8 8}$, 714-721.

65. Boespflug EL, McNamara RK, Eliassen JC, et al. (2016) Fish oil supplementation increases event-related posterior cingulate activation in older adults with subjective memory impairment. J Nutr Health Aging 20, 161-169.

66. Martinez-Lapiscina EH, Clavero P, Toledo E, et al. (2013) Virgin olive oil supplementation and long-term cognition: the PREDIMED-NAVARRA randomized trial. J Nutr Health Aging 17, 544-552.

67. Zamroziewicz MK \& Barbey AK (2016) Nutritional cognitive neuroscience: innovations for healthy brain aging. Front Neurosci 10, 240.

68. Lange KW, Lange KM, Makulska-Gertruda E, et al. (2017) Ketogenic diets and Alzheimer's disease. Food Sci Human Wellness 6, 1-9.

69. Gillette-Guyonnet S \& Vellas B (2008) Caloric restriction and brain function. Curr Opin Clin Nutr Metab Care 11, 686-692.

70. Luciano M, Corley J, Cox SR, et al. (2017) Mediterranean-type diet and brain structural change from 73 to 76 years in a Scottish cohort. Neurology 88, 449-455. 
71. Gu Y, Brickman AM, Stern Y, et al. (2015) Mediterranean diet and brain structure in a multiethnic elderly cohort. Neurology 85, 1744-1751.

72. Gardener H, Scarmeas N, Gu Y, et al. (2012) Mediterranean diet and white matter hyperintensity volume in the Northern Manhattan Study. Arch Neurol 69, 251-256.

73. Forlenza OV, Diniz BS, Teixeira AL, et al. (2015) Lower cerebrospinal fluid concentration of brain-derived neurotrophic factor predicts progression from mild cognitive impairment to Alzheimer's disease. Neuromol Med 17, 326-332.

74. Barberger-Gateau P (2014) Nutrition and brain aging: how can we move ahead? Eur J Clin Nutr 68, 1245-1249.

75. de Jager CA \& Kovatcheva A (2010) Summary and discussion: methodologies to assess long-term effects of nutrition on brain function. Nutr Rev 68, S53-S58.

76. Mosconi L \& McHugh PF (2015) Let food be thy medicine: diet, nutrition, and biomarkers' risk of Alzheimer's disease. Curr Nutr Rep 4, 126-135.

77. Misra S (2012) Randomized double blind placebo control studies, the "Gold Standard" in intervention based studies. Indian J Sex Transm Dis 33, 131-134.

78. Ferry M, Coley N, Andrieu S, et al. (2013) How to design nutritional intervention trials to slow cognitive decline in apparently healthy populations and apply for efficacy claims: a statement from the International Academy on Nutrition and Aging Task Force. J Nutr Health Aging 17, 619-623.

79. Katsiardanis K, Diamantaras AA, Dessypris N, et al. (2013) Cognitive impairment and dietary habits among elders: the Velestino Study. J Med Food 16, 343-350.

80. Portet F, Ousset PJ, Visser PJ, et al. (2006) Mild cognitive impairment (MCI) in medical practice: a critical review of the concept and new diagnostic procedure. Report of the MCI Working Group of the European Consortium on Alzheimer's Disease. J Neurol Neurosurg Psychiatry 77, 714-718.

81. Hughes CP, Berg L, Danziger WL, et al. (1982) A new clinical scale for the staging of dementia. Br J Psychiatry 140, 566-572.

82. Petersen RC. (2004) Mild cognitive impairment as a diagnostic entity. J Intern Med 256, 183-194.

83. Winblad B, Palmer K, Kivipelto M, et al. (2004) Mild cognitive impairment: beyond controversies, towards a consensus: report of the International Working Group on Mild Cognitive Impairment. J Intern Med 256, 240-246.

84. Petersen RC, Smith GE, Waring SC, et al. (1999) Mild cognitive impairment: clinical characterization and outcome. Arch Neurol 56, 303-308.

85. Dubois B, Feldman HH, Jacova C, et al. (2007) Research criteria for the diagnosis of Alzheimer's disease: revising the NINCDS-ADRDA criteria. Lancet Neurol 6, 734-746. 